\title{
Horizontal soil water potential heterogeneity: simplifying approaches for crop water dynamics models
}

\author{
V. Couvreur ${ }^{1,2}$, J. Vanderborght ${ }^{3}$, L. Beff ${ }^{1}$, and M. Javaux ${ }^{1,3}$ \\ ${ }^{1}$ Earth and Life Institute, Université catholique de Louvain, Croix du Sud, 2, bte L7.05.02, 1348 Louvain-la-Neuve, Belgium \\ ${ }^{2}$ Department of Land, Air and Water Resources, University of California, 1 Shields Ave., Davis, CA 95616, USA \\ ${ }^{3}$ Institute of Bio- und Geosciences, IBG-3: Agrosphere, Forschungszentrum Juelich GmbH, 52425 Juelich, Germany
}

Correspondence to: V. Couvreur (vcouvreur@ucdavis.edu)

Received: 2 January 2014 - Published in Hydrol. Earth Syst. Sci. Discuss.: 23 January 2014

Revised: - Accepted: 23 March 2014 - Published: 12 May 2014

\begin{abstract}
Soil water potential (SWP) is known to affect plant water status, and even though observations demonstrate that SWP distribution around roots may limit plant water availability, its horizontal heterogeneity within the root zone is often neglected in hydrological models. As motive, using a horizontal discretisation significantly larger than one centimetre is often essential for computing time considerations, especially for large-scale hydrodynamics models. In this paper, we simulate soil and root system hydrodynamics at the centimetre scale and evaluate approaches to upscale variables and parameters related to root water uptake (RWU) for two crop systems: a densely seeded crop with an average uniform distribution of roots in the horizontal direction (winter wheat) and a wide-row crop with lateral variations in root density (maize). In a first approach, the upscaled water potential at soil-root interfaces was assumed to equal the bulk SWP of the upscaled soil element. Using this assumption, the 3-D high-resolution model could be accurately upscaled to a 2-D model for maize and a 1-D model for wheat. The accuracy of the upscaled models generally increased with soil hydraulic conductivity, lateral homogeneity of root distribution, and low transpiration rate. The link between horizontal upscaling and an implicit assumption on soil water redistribution was demonstrated in quantitative terms, and explained upscaling accuracy. In a second approach, the soil-root interface water potential was estimated by using a constant rate analytical solution of the axisymmetric soil water flow towards individual roots. In addition to the theoretical model properties, effective properties were tested in order to account for unfulfilled assumptions of the analytical solution: non-uniform lateral root distributions and transient RWU rates. Significant im-
\end{abstract}

provements were however only noticed for winter wheat, for which the first approach was already satisfying. This study confirms that the use of 1-D spatial discretisation to represent soil-plant water dynamics is a worthy choice for densely seeded crops. For wide-row crops, e.g. maize, further theoretical developments that better account for horizontal SWP heterogeneity might be needed in order to properly predict soil-plant hydrodynamics in 1-D.

\section{Introduction}

Even though soil water potential (SWP) is known to affect plant water status, and more specifically plant actual transpiration rate $\left(T_{\mathrm{act}}\right)$, its horizontal variability within the root zone is neglected in many hydrological models, because of computational efficiency considerations and limitations in the actual monitoring of SWP with high spatial resolution (Beff et al., 2013).

In first-generation land surface schemes, the soil compartment was considered as a spatially homogeneous bucket, filled by precipitation and emptied by evapotranspiration (Manabe, 1969). This approach to plant water availability is considered as a "bulk approach", since the total amount of water in the soil bucket defines its water potential, independently of how water is distributed in the compartment. Later, a vertical discretisation of soil in multiple layers was considered. Root water uptake rates were proportional to relative root length densities and were affected by the water potential in each soil layer (Feddes et al., 1976). This approach allowed explicitly considering vertical capillary water fluxes 
in the soil and root distribution to evaluate plant water availability. However, the relation between the uptake and local water availability that is used in these models does either not consider the connectivity of the root system or uses rather ad hoc approaches to account for compensation of uptake from regions with a higher water availability (Javaux et al., 2013). Recent developments of models explicitly accounting for three-dimensional (3-D) SWP heterogeneity and water flow in the root system's hydraulic architecture (HA) (Doussan et al., 2006; Javaux et al., 2008) allowed investigating how plant water availability could be inferred from root system hydraulic properties and SWP distribution.

Based on the HA approach, a physically based macroscopic root water uptake (RWU) model, whose three plantscale parameters can be derived from root segment-scale hydraulic parameters distributed along root system architectures of any complexity, was developed by Couvreur et al. (2012). Since this model provides a 3-D solution of water flow from soil-root interfaces to plant collar, it needs to operate coupled to a 3-D "centimetre-scale" soil water flow model, which drastically increases the computational effort for soil-plant water flow simulations.

In the literature, one can find two contrasting conjectures that allow reducing the computing time by upscaling smallscale 3-D water flow models: (i) neglecting horizontal variations of SWP at the microscopic scale and using a coarser horizontal-scale discretisation to account for lateral fluxes that may be relevant at a larger scale, or (ii) using analytical approaches to account for microscopic gradients of SWP between the bulk soil and the soil-root interface.

By using a coarse discretisation of the soil domain, the first approach assumes that SWP is horizontally homogeneous in zones possibly ranging from the centimetre scale to the plant scale. This configuration most probably occurs under low climatic demand for water, in homogeneously rooted soils with high hydraulic conductivity (Schroeder et al., 2009b).

The second approach relies on a radial axisymmetric expression of the Richards equation around a single root. Approximate analytical solutions of water flow can be obtained by assuming a constant soil hydraulic conductivity or diffusivity (Gardner, 1960), or constant-rate water uptake by roots (Van Noordwijk and De Willigen, 1987; De Jong Van Lier et al., 2006; Schroeder et al., 2007, 2009a). When considering a regular distribution of roots in each soil layer, this approach can be used to create a 1-D RWU model, implicitly accounting for horizontal soil water flow (Raats, 2007; De Jong Van Lier et al., 2008; Jarvis, 2011). Yet, the simplifying assumptions of this approach may be constraining. In reality, local uptake is not at constant rate, but highly variable on a daily basis, notably due to variations of plant transpiration (Jolliet and Bailey, 1992; Sperling et al., 2012). In addition, differences in root hydraulic properties between different root types and horizontal heterogeneity of root density may lead to biased predictions of RWU when homo- geneously distributed roots with similar hydraulic properties are assumed (Schneider et al., 2010; Durigon et al., 2012).

The objective of this paper is to provide a theoretical framework and an exploratory analysis of methods aiming at simplifying horizontal soil water flow calculation within the root zone, for soil-plant water flow models. Therefore, an approach to upscale the macroscopic RWU model that was derived based on the fully discretised hydraulic root architecture by Couvreur et al. (2012) will be presented. The upscaling approach corresponding to the first conjecture will be tested under different conditions regarding atmospheric demand, soil type and rooting heterogeneity, so as to discuss its applicability field. The opportunities and obstacles tied to the second conjecture will be analysed in the last part.

\section{Theory}

When a soil system at hydrostatic equilibrium is impacted by external processes, like evaporation, transpiration or aquifer level rise, the uniform SWP distribution is perturbed. Internal fluxes like soil capillary fluxes, drainage and hydraulic lift, driven by SWP heterogeneity then come into play to dissipate this heterogeneity and stabilise the system to another equilibrium state, unless other external perturbations arise in the meantime. The resulting system state heterogeneity may hinder the accuracy of its upscaled representation. Such accuracy thus highly relies on system properties influencing the rates of processes generating and dissipating heterogeneity.

In this section, we present soil- and plant-water flow equations that generate and dissipate SWP heterogeneity.

\subsection{Equations for three-dimensional explicit water flow simulation}

Soil water capillary flow is driven by local gradients of SWP and tends to dissipate SWP heterogeneity. In this study, we assume 3-D soil water flow to be well described by the Richards equation:

$$
\frac{\partial \theta}{\partial t}=\nabla \cdot\left[K \nabla \psi_{\mathrm{s}}\right]-S,
$$

where $\theta$ is the volumetric water content $\left(\mathrm{L}^{3} \mathrm{~L}^{-3}\right), t$ is time (T), $K$ is the unsaturated soil hydraulic conductivity $\left(\mathrm{L}^{2} \mathrm{P}^{-1} \mathrm{~T}^{-1}\right)$ here considered as isotropic, $\psi_{\mathrm{s}}$ is the SWP $(\mathrm{P})$ including matric and gravimetric components of water potential, and $S$ is the sink term $\left(\mathrm{L}^{3} \mathrm{~L}^{-3} \mathrm{~T}^{-1}\right)$, which accounts for RWU. Note that the units of $K$ and $\psi_{\mathrm{s}}$ differ from standards of soil physics (in which $\mathrm{LT}^{-1}$ and $\mathrm{L}$ are more commonly used for $K$ and $\psi_{\mathrm{s}}$, respectively) but were chosen for consistency with those used in plant physiology.

In fine soil elements, the macroscopic RWU model based on the HA approach proposed by Couvreur et al. (2012) provides an expression for sink terms of the Richards equation: 
$S_{k} \cdot V_{k}=T_{\text {act }} \cdot \operatorname{SSF}_{k}+K_{\text {comp }} \cdot\left(\psi_{\mathrm{s}, k}-\psi_{\mathrm{seq}}\right) \cdot \mathrm{SSF}_{k}$,

where $S_{k}\left(\mathrm{~T}^{-1}\right)$ is the sink term in the $k$ th soil element, $V_{k}$ $\left(\mathrm{L}^{3}\right)$ is the volume of the $k$ th soil element, $T_{\text {act }}\left(\mathrm{L}^{3} \mathrm{~T}^{-1}\right)$ is the plant's actual transpiration rate, $\mathrm{SSF}_{k}(-)$ is the standard sink fraction in the $k$ th soil element (the sum of these individual fractions being one by definition), $K_{\text {comp }}\left(\mathrm{L}^{3} \mathrm{P}^{-1} \mathrm{~T}^{-1}\right)$ is the compensatory RWU conductance of the plant, $\psi_{\mathrm{s}, k}(\mathrm{P})$ is the SWP of the $k$ th soil element, and $\psi_{\mathrm{s} \text { eq }}(\mathrm{P})$ is the equivalent SWP sensed by the plant, which is a function of local SWPs and of the standard sink fraction distribution:

$\psi_{\mathrm{s} \text { eq }}=\sum_{j=1}^{M} \psi_{\mathrm{s}, j} . \mathrm{SSF}_{j}$,

where the $j$ index ranges from the first to the last of the $M$ soil elements $\left(\mathrm{SSF}_{j}\right.$ being zero for soil elements that do not contain any root segment).

Equations (2) and (3) rely on the assumption that the water potentials at soil-root interfaces located inside a soil element equal the element bulk SWP $\psi_{\mathrm{s}, k}$. If sufficiently small soil elements are used, this assumption may be satisfied (Schroeder et al., 2009a, b). Another simplifying assumption that needs to be fulfilled for Eq. (2) to be valid is that root radial conductances should be much lower than root axial conductances.

Equation (2) provides a conceptual split of the RWU variable into a "standard RWU" $\left(T_{\text {act }} \cdot \mathrm{SSF}_{k}\right)$ and a "compensatory RWU" $\left(K_{\mathrm{comp}} \cdot\left(\psi_{\mathrm{s}, k}-\psi_{\mathrm{s} \text { eq }}\right) \cdot \mathrm{SSF}_{k}\right)$. While the former creates SWP heterogeneity as long as the plant transpires, the latter is driven by, and tends to dissipate, SWP heterogeneity as long as SWP heterogeneity exists in the rooting zone.

With the HA approach, a link between water potential in the soil, at the plant collar, and actual transpiration rate is also provided by Couvreur et al. (2012):

$\psi_{\text {collar }}=\psi_{\mathrm{s} \mathrm{eq}}-\frac{T_{\mathrm{act}}}{K_{\mathrm{rs}}}$,

where $K_{\mathrm{rs}}\left(\mathrm{L}^{3} \mathrm{P}^{-1} \mathrm{~T}^{-1}\right)$ is the equivalent conductance of the root system, and $\psi_{\text {collar }}(\mathrm{P})$ is the water potential in xylem vessels at the plant collar, which will be referred to as the "plant collar water potential".

It is worth noting that, through Eq. (4), plant collar water potential can be interpreted as being the sum of the equivalent SWP sensed by the plant and of the water potential loss due to water flow in the root system.

The pathway of water from plant collar xylem vessels to leaves is considered as one of the least resistive from a hydraulic perspective, the main resistances being located in soil (Draye et al., 2010), between soil and root xylem (Frensch and Steudle, 1989), and between the inner leaf and atmosphere. For the purpose of simplification, we considered the hydraulic resistance from plant collar to leaves to be negligible as compared to the root system's hydraulic resistance. This is equivalent to assuming leaf water potential as equal to $\psi_{\text {collar }}$. By using Eq. (4), one can then estimate the plant transpiration rate from leaf water potential under water stress, $\psi_{\text {leaf stress }}(\mathrm{P})$ :

$T_{\text {water stress }}=K_{\text {rs }} \cdot\left(\psi_{\text {s eq }}-\psi_{\text {leaf stress }}\right)$,

where $T_{\text {water stress }}\left(\mathrm{L}^{3} \mathrm{~T}^{-1}\right)$ is the plant transpiration rate under water stress, and $\psi_{\text {leaf stress }}$ is a constant for isohydric plants such as maize (Tardieu and Simonneau, 1998).

The assumption on collar to leaf hydraulic resistance may however be inappropriate for certain types of plants (Domec and Pruyn, 2008), in which case the whole plant conductance should be used instead of $K_{\mathrm{rs}}$. Also, processes such as cavitation or aquaporin gating were not accounted for in this study, but may affect the plant conductance. Future prospects may concentrate on these aspects.

Considering that $T_{\text {act }}$ neither exceeds the plant's potential transpiration rate nor $T_{\text {water stress, }}$, we obtain the following simplistic water stress function:

$T_{\text {act }}=\min \left(T_{\text {pot }}, T_{\text {water stress }}\right)$,

where $T_{\text {pot }}\left(\mathrm{L}^{3} \mathrm{~T}^{-1}\right)$ is the plant's potential transpiration rate, which depends on both atmospheric conditions and plant leave properties.

It is worth noting that the variables and parameters presented in this section are representative for a single plant. They could also be used to obtain the average transpiration rate of several plants under water stress having the same $K_{\mathrm{rs}}$ (average $\psi_{\text {leaf stress }}$ and $\psi_{\mathrm{s} \text { eq }}$ then apply). However, as soon as the considered plants have significantly different $K_{\mathrm{rs}}$, such averaging method might not provide accurate estimates of average transpiration rate, and plants should be considered individually.

\subsection{Upscaling of water flow parameters and state variables}

\subsubsection{Plant water flow}

Equation (2) was set up for 3-D soil-plant water dynamics modelling on small soil elements (centimetre scale). Understanding the implications of its application to larger elements requires the definition of upscaled variables in terms of the original "fine-scale" variables and parameters $\left(S_{k}, V_{k}, \mathrm{SSF}_{k}\right.$ and $\psi_{\mathrm{s}, k}$ ). Here, we consider that upscaled soil elements are groups of smaller soil elements.

Since soil element volumes and standard sink fractions are extensive entities (i.e. additive for independent subsystems), their value for a group of soil elements is the sum of the soil elements values:

$V_{\mathrm{Up}, g}=\sum_{k=1}^{M} \varepsilon_{k, g} . V_{k}$, 
$\mathrm{SSF}_{\mathrm{Up}, g}=\sum_{k=1}^{M} \varepsilon_{k, g} \cdot \mathrm{SSF}_{k}$

where $V_{\mathrm{Up}, g}\left(\mathrm{~L}^{3}\right)$ is the "upscaled" volume of the $g$ th group, $\mathrm{SSF}_{\mathrm{Up}, g}(-)$ is the standard sink fraction of the gth group, $\varepsilon_{k, g}(-)$ is one when the $k$ th element belongs to the $g$ th group and zero otherwise, and the $k$ index ranges from the first to the last of the $M$ soil elements. Note that groups are nonoverlapping, so that the summation of $\mathrm{SSF}_{\mathrm{Up}, g}$ on the whole soil domain is 1, like for $\mathrm{SSF}_{k}$.

The sink term only becomes an extensive variable when multiplied by the associated soil element volume (then it becomes an additive flux). We can thus write

$S_{\mathrm{Up}, g} \cdot V_{\mathrm{Up}, g}=\sum_{k=1}^{M} \varepsilon_{k, g} \cdot S_{k} \cdot V_{k}$,

where $S_{\mathrm{Up}, g}\left(\mathrm{~T}^{-1}\right)$ is the sink term in the $g$ th group.

Upscaling the left and right hand sides of Eq. (2) leads to

$S_{\mathrm{Up}, g} \cdot V_{\mathrm{Up}, g}=T_{\mathrm{act}} \cdot \mathrm{SSF}_{\mathrm{Up}, g}+K_{\mathrm{comp}} \cdot\left(\psi_{\mathrm{sr} \mathrm{Up}, g}-\psi_{\mathrm{s} \text { eq }}\right)$

.SSFup, $g$.

From Eqs. (2) and (8)-(10), the upscaled soil-root interface water potential, $\psi_{\mathrm{sr} \mathrm{Up}, g}(\mathrm{P})$, is defined as

$\psi_{\mathrm{sr} \mathrm{Up}, g}=\frac{\sum_{k=1}^{M} \varepsilon_{k, g} \cdot \psi_{\mathrm{s}, k} \cdot \mathrm{SSF}_{k}}{\sum_{k=1}^{M} \varepsilon_{k, g} \cdot \mathrm{SSF}_{k}}$.

According to Eq. (11), the upscaled soil-root interface water potential represents the SSF-weighted mean SWP of the individual soil elements that constitute the upscaled soil element.

It is worth noting that the upscaled soil-root interface water potential $\psi_{\text {sr Up }}$ represents the SWP sensed by the plant in a part of the root zone. When it comprises the entire root zone, $\psi_{\text {sr Up }}$ is the plant sensed SWP $\psi_{\text {s eq }}$ (Eq. 3).

So as to illustrate this concept, three simple examples are shown in Fig. 1. In the first example, only soil element \# 3 contains a root segment. Following Eq. (11), $\psi_{\mathrm{sr}}$ Up,1 should equal the SWP of element \# 3. In other words, in group \# 1, the root segment only senses the SWP of element \# 3, which is its direct environment. In the second example, each soil element contains a root segment. Considering all non-null

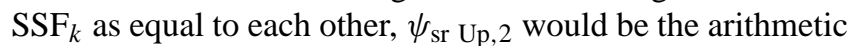
mean of the three individual SWPs. In the third example, no soil element contains a root segment so that $\mathrm{SSF}_{\mathrm{Up}, 3}$ is zero and no water potential sensed by root segments needs to be calculated for this element.

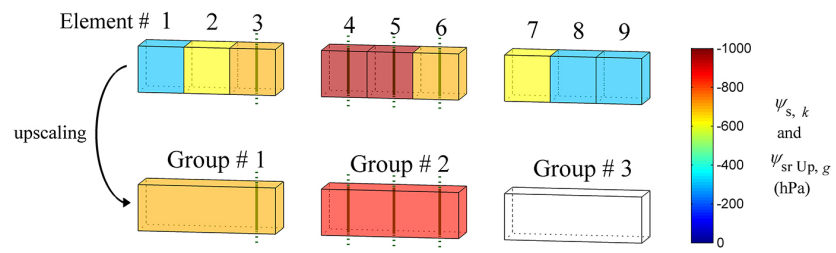

Fig. 1. Examples of the relation between $\psi_{\mathrm{s}, k}$ and $\psi_{\mathrm{sr} \text { Up, } g}$. Cubes are soil elements whose SWP, $\psi_{\mathrm{s}, k}$, is represented by the colour scale. Parallelepipeds are groups of three, upscaled, soil elements, whose upscaled soil-root interface water potential $\psi_{\mathrm{sr} \text { Up, } g}$ is represented by the same colour scale. Green vertical lines, in elements 3-6 and groups 1 and 2, are root segments.

Eventually, by using Eqs. (3), (8) and (11), it can be demonstrated that the equivalent SWP sensed by the plant can be calculated from $\mathrm{SSF}_{\mathrm{Up}}$ (vector size: $[G \times 1]$ ) using

$\psi_{\mathrm{s} \text { eq }}=\sum_{f=1}^{G} \psi_{\mathrm{sr} \mathrm{Up}, f} \cdot \mathrm{SSF}_{\mathrm{Up}, f}$,

where the $f$ index ranges from the first to the last of the $G$ groups of soil elements. The equations that are used to determine the plant-sensed soil water content (Eqs. 3, 12) and the local water uptake (Eqs. 2,10) are scale invariant, which follows directly from the fact that these relations are linear at the small scale. Similarly, the water stress equations (Eqs. 5, 6) are scale invariant and do not depend on the scale at which SSF and $\psi_{\text {sr }}$ are defined. A problem though is that for the calculation of the upscaled soil-root interface water potentials, $\psi_{\text {sr Up }}$, using Eq. (11) the distribution of the SWPs and SSF at the smaller scale must be known. In the following, we will make two assumptions to derive $\psi_{\text {sr Up }}$ directly from simulated upscaled SWPs and upscaled SSF.

\subsubsection{Soil water flow}

In this study, soil water flow state variables of upscaled elements were estimated with a simple "bulk" approach (i.e. the distribution of water inside upscaled soil elements was not accounted for). Their SWP, $\psi_{\mathrm{s} \text { Up }}(\mathrm{P})$, and hydraulic conductivity, $K_{\mathrm{Up}}\left(\mathrm{L}^{2} \mathrm{P}^{-1} \mathrm{~T}^{-1}\right)$, were directly deduced from their bulk water content $\theta_{\mathrm{Up}}\left(\mathrm{L}^{3} \mathrm{~L}^{-3}\right)$ and, respectively, water retention curve and hydraulic conductivity curve (these properties being uniform in space and time).

In consequence, the following upscaled expression of the Richards equation was used:

$\frac{\partial \theta_{\mathrm{Up}}}{\partial t}=\nabla \cdot\left[K_{\mathrm{Up}} \nabla \psi_{\mathrm{s} \mathrm{Up}}\right]-S_{\mathrm{Up}}$,

where $S_{U p}$ is provided by Eq. (10). 


\subsection{Simplifying assumptions for horizontal soil water flow}

\subsubsection{First conjecture: homogeneous soil water potential in upscaled soil elements}

In simulations with upscaled soil elements (for instance in a 1-D soil domain), detailed SWPs around individual root segments are not available. In the first proposed approach, upscaled soil-root interface water potentials were approximated by the corresponding element bulk SWP:

$\psi_{\mathrm{sr} \mathrm{Up}, g}=\psi_{\mathrm{m}}\left(\theta_{\mathrm{Up}, g}\right)+z_{g}$,

where $\psi_{\mathrm{m}}(\theta)(\mathrm{P})$ is the function providing soil matric potential from soil water content, $\theta_{\mathrm{Up}, g}\left(\mathrm{~L}^{3} \mathrm{~L}^{-3}\right)$ is the bulk water content of the $g$ th upscaled soil element, and $z_{g}(\mathrm{P})$ is the gravitational potential of water at the centre of the $g$ th upscaled soil element. Note that $z_{g}$ is defined zero at the soil surface and positive upwards.

This assumption is generally considered as consistent either on short distances (as in fine elements of reference scenarios), or in conditions of high soil hydraulic conductivity (when lateral redistribution of water occurs almost instantaneously).

When water is redistributed by soil capillary flow (or by compensatory RWU), a positive divergence of water flow is generated at points where water is removed, while a negative divergence occurs where water is added. Considering water mass conservation, the volumetric integration of positive water divergences related to the process of redistribution must equal the volumetric integration of negative water divergences. Both integrated terms represent a volume of water moved from a place to another one per time unit, and equal a rate of water redistribution.

By assuming SWP as permanently homogeneous in an environment where water uptake is actually local, it is implicitly hypothesised that the divergence of soil water flow is high enough to instantly compensate for the removal of water by roots. For a given uptake rate in an upscaled element, and knowing the fine distribution of the standard sink fractions inside the element, it can be demonstrated (see Appendix A) that the soil water redistribution rate required to maintain SWP homogeneous inside the element should be the following:

$R_{\text {Soil } \leftrightarrow \text { hyp }, g}=\left|S_{\mathrm{Up}, g}\right| \cdot V_{\mathrm{Up}, g} \cdot \frac{\sum_{k=1}^{M} \varepsilon_{k, g} \cdot\left|\frac{\mathrm{SSF}_{k}}{\mathrm{SSF}_{\mathrm{Up}, g}}-\frac{V_{k}}{V_{\mathrm{Up}, g}}\right|}{2}$,

where $R_{\text {soil } \leftrightarrow \text { hyp, } g}\left(\mathrm{~L}^{3} \mathrm{~T}^{-1}\right)$ is the soil water redistribution rate required in order to keep the SWP horizontally homogeneous in the $g$ th group of soil elements.

Note that soil water flow divergence at scales lower than the fine scale of the reference scenarios is not considered in the latter equation.

\subsubsection{Second conjecture: solution for implicit SWP horizontal heterogeneity in soil layers}

In the second proposed approach, the De Jong Van Lier et al. (2008) model provides a solution for differences between bulk soil and soil-root interface water potentials within 1-D soil elements, which does not require explicitly solving horizontal soil water flow. The latter is coupled to the upscaled macroscopic RWU model (Eq. 10), which simulates the consequent vertical water flow in root system HA.

The solution for horizontal soil water flow around roots relies on the concept of matric flux potential (MFP), which is the integral of soil hydraulic conductivity curve $K\left(\psi_{\mathrm{m}}\right)$, over soil matric potential $\psi_{\mathrm{m}}(\mathrm{P})$, and, equivalently, the integral of the soil diffusivity curve $D(\theta)\left(\mathrm{L}^{2} \mathrm{~T}^{-1}\right)$, over soil water content $\theta\left(\mathrm{L}^{3} \mathrm{~L}^{-3}\right)$ :

$M\left(\psi_{\mathrm{m}}, \theta\right)=\int_{\psi_{\mathrm{w}}}^{\psi_{\mathrm{m}}} K\left(\psi_{\mathrm{m}}\right) \cdot \mathrm{d} \psi_{\mathrm{m}}=\int_{\theta_{\mathrm{w}}}^{\theta} D(\theta) . \mathrm{d} \theta$,

where $M\left(\psi_{\mathrm{m}}, \theta\right)\left(\mathrm{L}^{2} \mathrm{~T}^{-1}\right)$ is the soil MFP at soil matric potential $\psi_{\mathrm{m}}$ or soil water content $\theta, \psi_{\mathrm{w}}(\mathrm{P})$ is the soil matric potential at permanent wilting point, and $\theta_{\mathrm{W}}\left(\mathrm{L}^{3} \mathrm{~L}^{-3}\right)$ the soil water content at permanent wilting point.

By assuming root distribution as horizontally regular and the rate of uptake as constant, De Jong Van Lier et al. (2008) provide a simple relation between RWU rate in a soil layer $\left(S_{\mathrm{Up}, g}\right)$, bulk soil layer MFP $M_{\mathrm{S}} \mathrm{Up}, g\left(\mathrm{~L}^{2} \mathrm{~T}^{-1}\right)$, and MFP at soil-root interfaces in that soil layer $M_{\mathrm{sr}} \mathrm{Up}, g\left(\mathrm{~L}^{2} \mathrm{~T}^{-1}\right)$, which implicitly accounts for SWP horizontal heterogeneity:

$M_{\mathrm{sr} \mathrm{Up}, g}=M_{\mathrm{SUp}, g}-\frac{S_{\mathrm{Up}, g}}{\rho_{g}}$,

where $S_{\mathrm{Up}, g}$ is given by Eq. (10), and $\rho_{g}\left(\mathrm{~L}^{-2}\right)$ is a geometrical factor depending on rooting density and root radius at the $g$ th depth (see Eq. B1). The factor $\rho$ decreases with decreasing rooting density (and thus typically with depth). Decreasing $\rho$ or increasing sink terms induce larger differences between $M_{\mathrm{S} \mathrm{Up}, g}$ and predicted $M_{\mathrm{sr} \mathrm{Up}, g}$.

By using the MFP curve, which links a soil matric potential to its MFP, one can derive $\psi_{\mathrm{sr} \mathrm{Up}, g}$ from $M_{\mathrm{sr} \mathrm{Up}, g}$ :

$\psi_{\mathrm{sr} \mathrm{Up}, g}=\psi_{\mathrm{m}}\left(M_{\mathrm{sr} \mathrm{Up}, g}\right)+z_{g}$,

where $\psi_{\mathrm{m}}(M)(\mathrm{P})$ is the function providing soil matric potential from soil MFP.

As compared to Eq. (14), Eq. (18) is an alternative way to estimate soil-root interface's water potential in relatively large soil elements.

Knowing $\psi_{\mathrm{sr} \text { Up, } g}$ in every soil layer, the equivalent SWP sensed by the plant can be calculated (Eq. 12), which allows further calculations of the plant's actual transpiration (Eqs. 5, 6) and RWU distribution (Eq. 10). 


\section{Methodology}

So as to discuss up to what point the first soil water flow simplification leads to worthy compromises between accuracy and computing time, the conjecture of homogeneous SWP in upscaled soil elements was tested in different scenarios. These scenarios further described in Sect. 3.1 varied in (i) rooting heterogeneity, (ii) soil type, and (iii) atmospheric demand for water. Section 3.2 explains in detail the methods used to evaluate both conjectures implemented as options in R-SWMS (Root-Soil Water Movement and Solute transport; Javaux et al., 2008).

\subsection{Scenarios description}

\subsubsection{Root systems architecture and hydraulic properties}

Two crops with typically contrasting root distributions in the field were chosen for this study.

The first one is maize, whose horizontal rooting density varies more in the perpendicular direction than in the parallel direction to the row, due to its "wide row" sowing pattern (here corresponding to $75 \mathrm{~cm} \times 15 \mathrm{~cm}$ ). The generation and parameterisation of the 80 days-old virtual maize root system used in this study is fully described by Couvreur et al. (2012).

The second crop is winter wheat, whose horizontal rooting density is more homogeneous than that of maize, due to a dense seeding pattern. A density of 140 plants $\mathrm{m}^{-2}$ with a distance between plants of $10 \mathrm{~cm}$ in the $x$ direction and $7 \mathrm{~cm}$ in the $y$ direction was considered.

A winter wheat root system at early spring of 17000 segments was generated with RootTyp (Pages et al., 2004). This model generates root systems based on plant-specific genetic properties like insertion angles of the different root types, their trajectories, average growth speed and distances between lateral roots, which were characterised for a winter wheat during early spring, in Nebraska (USA), by Weaver et al. (1924). They were also used to adapt RootTyp environmental parameters so as to reproduce measured root-length density profiles. The optimised wheat root system architecture is shown in Fig. 2a.

Wheat root's hydraulic properties were dependent on root segment age and type (shown in Fig. 2b and c) and were obtained from the literature. Root segments radial conductivities were measured by Tazawa et al. (1997) and Bramley et al. (2007, 2009). Root segment's axial conductance were measured by Sanderson et al. (1988) and Bramley et al. (2007) for primary roots, while Watt et al. (2008) estimated this property for lateral roots by using the Poiseuille-Hagen law.

So as to represent winter wheat root distribution in the field and accounting for the effect of overlapping root zones from neighbouring plants, while limiting the computational needs, the virtual root system was located in a horizontally periodic soil domain of $10 \mathrm{~cm} \times 7 \mathrm{~cm}$, which corresponds to the spacing between plants. Periodicity was applied for root system architecture at the vertical boundaries of the domain. Viewed from a larger scale than the individual plant scale, this case would correspond to a field containing identical root system architectures regularly spaced. In consequence, SWP variability is only accounted for at scales lower or equal to the plant scale.

\subsubsection{Soil hydraulic properties}

Two soil types with typically contrasting hydraulic properties were chosen for this study. The first one is a silt loam, whose water capacity and hydraulic conductivity are relatively high for a wide range of soil matric potentials (properties represented in blue, respectively in Fig. 3a and b).

The second soil type is a sandy loam, whose hydraulic conductivity is quite high close to water saturation, but soon becomes resistive to water flow when SWP decreases (properties represented in red, respectively in Fig. 3a and b).

Note that Mualem-van Genuchten equations (Van Genuchten, 1980) were used to define the soil hydraulic property curves, and that Carsel and Parrish (1988) parameterisations were chosen for both soil types.

In the scenarios, SWP was initially uniform (hydrostatic equilibrium) and set to field capacity $(-300 \mathrm{hPa})$ for the silt loam. Sandy loam initial water potential was set to $-130 \mathrm{hPa}$, so that water availability would not be limiting the uptake during the first days of the scenarios.

The soil domain was $123 \mathrm{~cm}$ deep, which means that for an initially uniform SWP, and neglecting the effect of osmotic potential, there was a difference of approximately $123 \mathrm{hPa}$ between top and bottom matric potentials. This implied that soil water content and hydraulic conductivity were changing along the soil profile, already at initial conditions, as illustrated by the coloured bands in Fig. 3 .

\subsubsection{Boundary conditions}

In order to focus on RWU and soil capillary flow as processes generating or reducing SWP heterogeneity, no other processes were considered in the scenarios. Therefore, noflux boundary conditions were set at the top and bottom boundaries of the soil domain, while plant transpiration was the only process removing water from the system. In addition to being periodic for the root system architecture, vertical boundaries of the domain were periodic for soil- and root-water flow.

High- and low-transpiration-rate cases were selected in order to investigate whether these rates impact the validity of simplifying assumptions about lateral SWP distributions in the root zone. Atmospheric demand for water reflected the geographical position and period of the year for which the root system architectures were determined. The FAO approach (Allen et al., 1998) was used to determine the daily 
a)

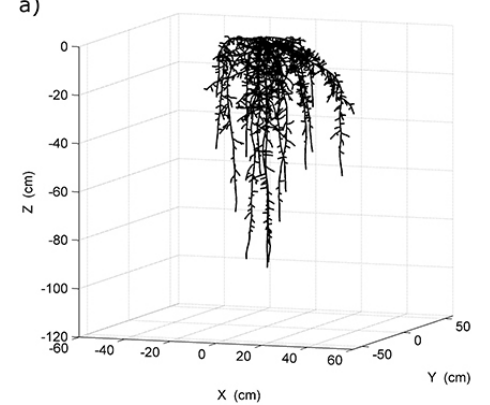

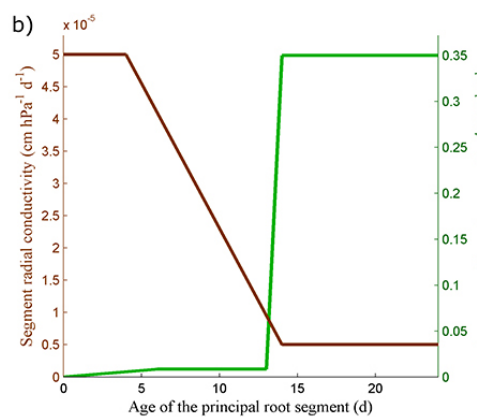

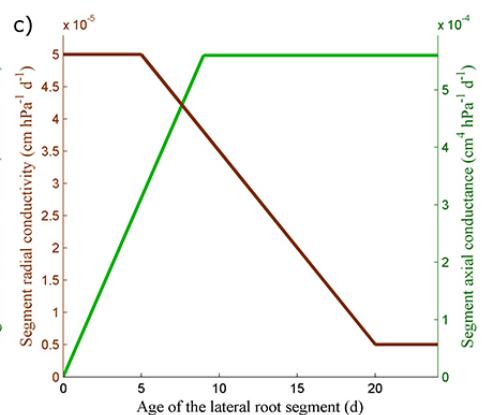

Fig. 2. Virtual winter wheat root system (a) architecture at early spring, and (b) principal and (c) lateral root segments' hydraulic properties.

a)
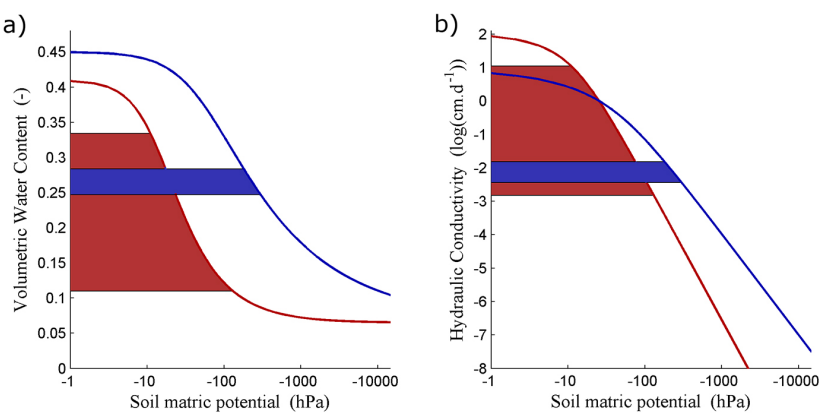

Fig. 3. Silt loam (blue) and sandy loam (red) hydraulic properties: (a) water retention curves and (b) hydraulic conductivity curves. The coloured bands show the ranges of (a) water content and (b) hydraulic conductivities initially met in the soil profile.

potential transpiration rate of single plants, $T_{\text {daily }}\left(\mathrm{L}^{3} \mathrm{~T}^{-1}\right)$, from selected reference evapotranspiration rates:

$T_{\text {daily }}=\mathrm{ET}_{\text {ref }} \cdot K_{\mathrm{c}} \cdot$ Surf,

where $\mathrm{ET}_{\text {ref }}\left(\mathrm{L} \mathrm{T}^{-1}\right)$ is the reference evapotranspiration, $K_{\mathrm{c}}$ $(-)$ is the crop coefficient, and $\operatorname{Surf}\left(\mathrm{L}^{2}\right)$ is the horizontal surface occupied by a single plant in a field. Note that the part of evaporation in $\mathrm{ET}_{\text {ref }}$ was considered as negligible. Accounting for it would have led to slightly lower transpiration rates.

For the French maize crop in July, $K_{\mathrm{c}}$ was 1.2 , Surf was $1125 \mathrm{~cm}^{2}$, and the high $\mathrm{ET}_{\text {ref }}$ was $4.5 \mathrm{~mm} \mathrm{~d}^{-1}$ while the low $\mathrm{ET}_{\text {ref }}$ was $2.25 \mathrm{~mm} \mathrm{~d}^{-1}$. For the Nebraskan winter wheat crop at early spring, $K_{\mathrm{c}}$ was 1 , Surf was $70 \mathrm{~cm}^{2}$, and the high $\mathrm{ET}_{\text {ref }}$ was $3.9 \mathrm{~mm} \mathrm{~d}^{-1}$ while the low $\mathrm{ET}_{\text {ref }}$ was $1.95 \mathrm{~mm} \mathrm{~d}^{-1}$.

Sinusoidal daily variations of $T_{\text {pot }}$ were expressed as a function of $T_{\text {daily }}$ with the following expression:

$T_{\text {pot }}=T_{\text {daily }} \cdot\left(\sin \left(\frac{2 \pi \cdot t}{\tau}-\frac{\pi}{2}\right)+1\right)$,

where $t(\mathrm{~T})$ is the time after midnight, and $\tau(\mathrm{T})$ is the number of time units in a day-night cycle (e.g. $\tau$ is $24 \mathrm{~h}$ if $t$ is given in hours, and 1 day if $t$ is given in days).

$\psi_{\text {leaf stress, which triggers stomata partial closure due }}$ to water stress (see Eqs. 5 and 6), was $-15000 \mathrm{hPa}$ for both crops.
The duration of scenarios is 14 days, except for high $\mathrm{ET}_{\text {ref }}$ on sandy loam (10 days).

\subsection{Testing the simplifying approaches}

The simplifying approaches described in Sect. 2.3 were tested by comparing their results with simulated reference results. In the reference simulations, Richards equation was solved for a fine 3-D soil grid, and the model of Doussan et al. (1998) was used to predict RWU by the root system HA in R-SWMS. Due to computing power considerations, the reference maize crop scenarios could not be run with soil elements smaller than cubes of $1.5 \mathrm{~cm}$ length. Since the winter wheat domain dimensions were smaller, its reference scenarios could be run with cubic soil elements of $0.5 \mathrm{~cm}$ length. Consequently, reference scenarios do not account for additional SWP gradients around roots at scales smaller than, respectively, 1.5 and $0.5 \mathrm{~cm}$. Accounting for this feature may increase differences between reference results and results obtained from upscaled soil grids (Schroeder et al., 2009b).

\subsubsection{Simplifying approaches features}

In order to test the first conjecture (homogeneous SWP in upscaled soil elements), each of the eight scenarios defined in Sect. 3.1 (combinations of the following properties: maize or winter wheat; silt loam or sandy loam; high or low $T_{\text {daily }}$ ) were run with soil elements of increasing horizontal surface, as summarised in Table 1 and illustrated in Fig. 4.

For maize, the assumption on SWP homogeneity was firstly applied to the direction parallel to the row. Subsequently, the discretisation was coarsened in the direction perpendicular to the rows. Therefore, all intermediate soil discretisations, between the finest one and 1-D, are 2-D (see Table 1). This is not the case for winter wheat, for which no preferential direction was considered to group soil elements.

In opposition, the second conjecture (soil-root interface water potential predicted from the approximate analytical solution of water flow towards a root) was directly tested for 1-D soil layers $(75 \mathrm{~cm} \times 15 \mathrm{~cm} \times 1.5 \mathrm{~cm}$ and 
Table 1. Sizes of upscaled soil elements and domain properties for both maize and winter wheat crops in the runs testing the first conjecture.

\begin{tabular}{llllllll}
\hline Plant type & Element properties & Case 1 & Case 2 & Case 3 & Case 4 & Case 5 & Case 6 \\
\hline \multirow{2}{*}{ Maize } & Horizontal area $\left(\mathrm{cm}^{2}\right)$ & 2.25 & 22.5 & 45 & 112.5 & 225 & 1125 \\
& $x$ and $y$ lengths $\left(\mathrm{cm}^{2}\right)$ & $1.5 \times 1.5$ & $1.5 \times 15$ & $4 \times 15$ & $7.5 \times 15$ & $15 \times 15$ & $75 \times 15$ \\
& Elements per layer (-) & 500 & 50 & 25 & 10 & 5 & 1 \\
& Domain dimensionality & 3 -D & $2-\mathrm{D}$ & 2 -D & 2 -D & 2-D & 1-D \\
\hline \multirow{2}{*}{ Winter wheat } & Horizontal area $\left(\mathrm{cm}^{2}\right)$ & 0.25 & 1 & 7 & 70 & & \\
& $x$ and $y$ lengths $(\mathrm{cm})$ & $0.5 \times 0.5$ & $1 \times 1$ & $2 \times 3.5$ & $10 \times 7$ & & \\
& Elements per layer (-) & 280 & 70 & 10 & 1 & & \\
& Domain dimensionality & 3-D & 3-D & 3-D & 1-D & & \\
\hline
\end{tabular}
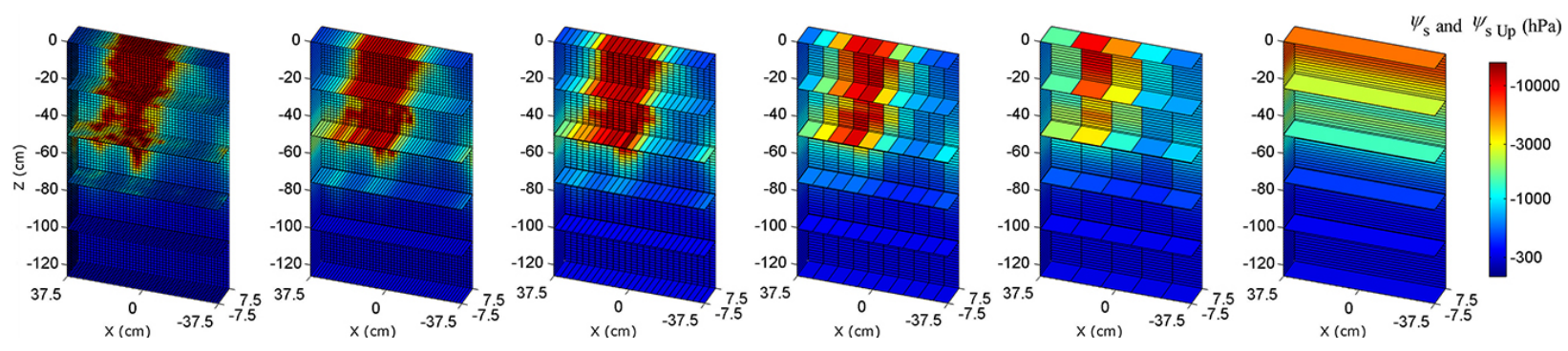

Fig. 4. Discretisations of the maize crop soil domain used for the first simplifying approach. The colour scale gives the soil water potential distribution at the end of the high-transpiration-rate scenario on silt loam.

$10 \mathrm{~cm} \times 7 \mathrm{~cm} \times 0.5 \mathrm{~cm}$, respectively for maize and winter wheat).

\subsubsection{Comparison with reference scenarios}

In order to evaluate the accuracy of the first simplified approach, differences between the reference and different upscaling scenarios were estimated for $\psi_{\text {collar }}$ and horizontally averaged sink term and water content profiles. The mean of the absolute differences for all times and depths was divided by the mean value for the reference case, which provided one relative mean absolute difference for each scenario. The relative computation time of the simplified to reference simulations was also determined.

Eventually, horizontal and vertical redistribution of water by both soil and roots from 1-D and reference results were compared, in order to understand which process dissipating SWP heterogeneity would be responsible of possibly wrong representations of 1-D soil-plant water dynamics. For simulations directly run in 1-D, the total horizontal redistribution of water by soil was estimated as the integration of the redistribution necessary to keep each layer's inner water potential homogeneous (i.e. vertical integration of Eq. 15). Other equations quantifying vertical and horizontal water redistribution by soil and roots from reference and 1-D simulation results are detailed in Appendix C.

With the second conjecture, simple effective methods that allow overcoming basic assumptions of the De Jong Van Lier et al. (2006) model were discussed. These concern (i) hor- izontal heterogeneity of root distribution, and (ii) transient rate of water uptake. For reasons discussed in Sect. 4.4.2, a proper coupling with the Richards equation could not be achieved with this conjecture. However, using bulk SWP data from the reference simulation and keeping past uptake rates in memory, we could evaluate the accuracy of the second conjecture at each individual time step.

Effective values of the geometrical parameter $\rho$ were first estimated from reference simulations and compared to theoretical values (calculated from each layer's root length density and assuming a regular distribution of roots), in order to understand how this parameter may be affected by horizontal rooting heterogeneity. Then, $\psi_{\mathrm{sr}} \mathrm{Up}$ was predicted from either the current sink term, or a weighted mean of sink terms on time windows of chosen length (weights linearly decreasing to zero with passed time), in order to understand if the history of past sink terms should be accounted for when RWU is transient.

Considering that the simplifying approaches presented in this paper introduce structural errors in the model, differences as compared to reference scenarios were considered as "errors". However, also the reference model is subject to structural errors (supposed relatively small). These basic errors were not accounted for in the next pages. 
Table 2. Relative absolute differences on $\psi_{\text {collar, }}$ 1-D sink terms, 1-D water contents and computing times in the maize scenarios, for increasing soil element sizes. Refer to Table 1 for the detailed geometry of cases 1-6.

\begin{tabular}{|c|c|c|c|c|c|c|c|}
\hline & \multirow[b]{2}{*}{ Maize scenario } & \multicolumn{6}{|c|}{ Case \# } \\
\hline & & 1 & 2 & 3 & 4 & 5 & 6 \\
\hline \multirow{4}{*}{$\begin{array}{l}\text { Relative difference } \\
\text { on } \psi_{\text {collar }}(\%)\end{array}$} & Low $T_{\text {daily }}-$ silt loam & 0.5 & 0.3 & 0.9 & 5.1 & 10.6 & 14.8 \\
\hline & High $T_{\text {daily }}-$ silt loam & 0.9 & 1.5 & 4.5 & 15.5 & 26.8 & 30.3 \\
\hline & Low $T_{\text {daily }}$ - sandy loam & 1.9 & 2.9 & 8.8 & 25.9 & 30.6 & 32.7 \\
\hline & High $T_{\text {daily }}$ - sandy loam & 3.7 & 4.6 & 8.1 & 13.2 & 15.0 & 18.7 \\
\hline \multirow{4}{*}{$\begin{array}{l}\text { Relative difference } \\
1-\mathrm{D} \text { sink }(\%)\end{array}$} & Low $T_{\text {daily }}-$ silt loam & 1.0 & 1.2 & 1.2 & 5.3 & 12.1 & 17.1 \\
\hline & High $T_{\text {daily }}-$ silt loam & 1.9 & 3.2 & 4.2 & 11.1 & 19.7 & 24.2 \\
\hline & Low $T_{\text {daily }}$ - sandy loam & 3.4 & 5.0 & 6.8 & 21.3 & 35.4 & 38.5 \\
\hline & High $T_{\text {daily }}-$ sandy loam & 6.3 & 8.0 & 10.9 & 24.3 & 44.9 & 47.4 \\
\hline \multirow{4}{*}{$\begin{array}{l}\text { Relative difference } \\
\text { 1-D water cont. (\%) }\end{array}$} & Low $T_{\text {daily }}-$ silt loam & 0.6 & 0.6 & 0.5 & 2.6 & 5.4 & 10.0 \\
\hline & High $T_{\text {daily }}-$ silt loam & 1.3 & 1.9 & 2.1 & 5.1 & 9.5 & 17.0 \\
\hline & Low $T_{\text {daily }}$ - sandy loam & 2.0 & 2.8 & 3.4 & 8.6 & 13.6 & 22.4 \\
\hline & High $T_{\text {daily }}-$ sandy loam & 2.4 & 4.4 & 5.1 & 9.3 & 14.2 & 22.8 \\
\hline \multirow{4}{*}{$\begin{array}{l}\text { Relative comput. } \\
\text { time }(\%)\end{array}$} & Low $T_{\text {daily }}-$ silt loam & 3.0 & 1.5 & 1.2 & 1.1 & 0.81 & 0.37 \\
\hline & High $T_{\text {daily }}-$ silt loam & 1.9 & 0.21 & 0.13 & 0.10 & 0.10 & 0.09 \\
\hline & Low $T_{\text {daily }}$ - sandy loam & 3.9 & 0.46 & 0.36 & 0.33 & 0.29 & 0.26 \\
\hline & High $T_{\text {daily }}-$ sandy loam & 0.98 & 0.04 & 0.03 & 0.02 & 0.02 & 0.02 \\
\hline
\end{tabular}

\section{Results and discussion}

\subsection{First conjecture: homogeneous soil water potential in upscaled soil elements}

Tables 2 and 3 show the relative errors of predicted state variables and relative computing time for each scenario, with increasing element size inside which SWP is assumed homogeneous. Errors that occur at the finest spatial discretisation (i.e. horizontal surfaces of respectively 2.25 and $0.25 \mathrm{~cm}^{2}$ for maize and winter wheat) are due to the replacement of the Doussan RWU model by Eq. (2) to calculate the sink terms.

It is notable that 1-D sink terms and $\psi_{\text {collar }}$ were generally more sensitive to errors than 1-D water contents, even though water content differences are a consequence of sink term differences. This can be explained by the fact that SWP heterogeneity is the driver of soil water flow. Thus, for instance, locally overestimating RWU leads to higher SWP heterogeneity, which leads to higher "compensation" by soil water flow. Consequently, errors of RWU tend to be larger than errors of soil water content, especially in cases of high soil hydraulic conductivity.

In the next sections, we study the impact of element size, daily transpiration rate and soil type on the reported relative absolute differences, and further analyse where these differences take place in space and time. Illustrations are mostly given for the scenario "high $T_{\text {daily }}$ on silt loam", but complementary explanations are given for other scenarios in case their trends differ from the illustrations.

\subsubsection{Impact of element size and crop type}

For maize, the simplification from 3-D to 2-D soil discretisation results in a relatively small increase of model errors (see Fig. 5a) since SWP is quite homogeneous in the direction of maize rows (see left subplot in Fig. 4). Conversely, further increases of element size in the direction perpendicular to maize rows (in which a big part of SWP variability is observed in reference scenarios) result in significant increase of model errors, particularly beyond case \#3 (elements of $3 \mathrm{~cm}$ in the direction perpendicular to the row). This result encourages the use of 2-D soil discretisation for simulating water dynamics in a maize crop, whereas considering a 1-D approach with homogeneous SWP in horizontal soil layers leads to strong errors in predicted state variables (approaching $50 \%$ of relative error on 1-D sink terms and $\psi_{\text {collar }}$ over a period of 10 days on sandy loam).

For winter wheat, while changing the element dimension from 3-D to 1-D (see Fig. 5b), model errors stayed remarkably low (below $1 \%$ for scenarios on silt loam over a period of 14 days). This feature can be related to the dense sowing pattern of the winter wheat crop (140 plants $\mathrm{m}^{-2}$, against 9 for maize), which naturally induces rather homogeneous horizontal rooting, uptake and SWP patterns.

One of the main interests of simplifying approaches is model computing time reduction. As shown in Table 2 (and illustrated in Fig. $5 \mathrm{c}$ and d), for maize, if computing time was already reduced by a factor of 25-100 due to the replacement of the Doussan model by Eq. (2), another factor of 3-30 was gained by using a 2-D soil discretisation. For winter wheat, 
Table 3. Relative absolute differences on $\psi_{\text {collar }}$, 1-D sink terms, 1-D water contents and computing times in the winter wheat scenarios, for increasing soil element sizes. Refer to Table 1 for the detailed geometry of cases 1-4.

\begin{tabular}{|c|c|c|c|c|c|}
\hline & \multirow[b]{2}{*}{ Winter wheat scenario } & \multicolumn{4}{|c|}{ Case \# } \\
\hline & & 1 & 2 & 3 & 4 \\
\hline \multirow{4}{*}{$\begin{array}{l}\text { Relative difference } \\
\text { on } H_{\text {collar }}(\%)\end{array}$} & Low $T_{\text {daily }}-$ silt loam & 0.0 & 0.0 & 0.1 & 0.1 \\
\hline & High $T_{\text {daily }}-$ silt loam & 0.1 & 0.2 & 0.5 & 0.6 \\
\hline & Low $T_{\text {daily }}-$ sandy loam & 0.3 & 1.8 & 2.5 & 2.5 \\
\hline & High $T_{\text {daily }}-$ sandy loam & 4.0 & 4.8 & 5.2 & 5.6 \\
\hline \multirow{4}{*}{$\begin{array}{l}\text { Relative difference } \\
1-\mathrm{D} \text { sink }(\%)\end{array}$} & Low $T_{\text {daily }}-$ silt loam & 0.2 & 0.2 & 0.2 & 0.2 \\
\hline & High $T_{\text {daily }}-$ silt loam & 0.4 & 0.5 & 0.7 & 0.8 \\
\hline & Low $T_{\text {daily }}$ - sandy loam & 0.9 & 2.9 & 4.6 & 4.9 \\
\hline & High $T_{\text {daily }}-$ sandy loam & 5.9 & 10.9 & 14.1 & 15.8 \\
\hline \multirow{4}{*}{$\begin{array}{l}\text { Relative difference } \\
\text { 1-D water cont. (\%) }\end{array}$} & Low $T_{\text {daily }}-$ silt loam & 0.1 & 0.1 & 0.1 & 0.1 \\
\hline & High $T_{\text {daily }}-$ silt loam & 0.1 & 0.2 & 0.3 & 0.3 \\
\hline & Low $T_{\text {daily }}$ - sandy loam & 0.19 & 1.1 & 2.0 & 2.4 \\
\hline & High $T_{\text {daily }}-$ sandy loam & 2.8 & 3.6 & 4.9 & 5.9 \\
\hline \multirow{4}{*}{$\begin{array}{l}\text { Relative comput. } \\
\text { time }(\%)\end{array}$} & Low $T_{\text {daily }}-$ silt loam & 6.9 & 4.8 & 1.9 & 1.5 \\
\hline & High $T_{\text {daily }}$ - silt loam & 9.0 & 3.3 & 1.5 & 0.98 \\
\hline & Low $T_{\text {daily }}-$ sandy loam & 17 & 3.6 & 1.3 & 0.79 \\
\hline & High $T_{\text {daily }}$ - sandy loam & 11 & 1.1 & 0.27 & 0.10 \\
\hline
\end{tabular}

using Eq. (2) only reduced computing time by a factor of 6-14 because its root system has half of the segments than maize (using Doussan model is computationally cheaper for small root systems, while computing time of Eq. (2) does not discriminate between big and small root systems). Computation time was reduced by another factor of 5-100 as compared to the high resolution 3-D winter wheat scenarios, by using 1-D soil elements.

Such results suggest that using the first conjecture in, respectively, 2-D (maize) and 1-D (winter wheat) soil elements as simplifying hypothesis for SWP distribution, is a worthy compromise maintaining accuracy while reducing computation time.

\subsubsection{Impact of daily transpiration and soil type}

Even though crop type and soil elements size had major impact on the simplifying approach accuracy, two other features also clearly impacted this accuracy: $T_{\text {daily }}$ and soil type.

Almost systematically, the simplified model accuracy was higher when decreasing $T_{\text {daily, }}$, and in the silt loam than in the sandy loam. Since accuracy under the first conjecture is highly related to the absence of SWP horizontal heterogeneity, the previous statement can be explained through processes involving creation and dissipation of SWP heterogeneity.

Firstly, standard RWU is a process creating SWP heterogeneity in a soil with an initial hydrostatic equilibrium state; increasing $T_{\text {daily }}$ (and obviously standard RWU) will thus lead to increased SWP heterogeneity and decreased accuracy under the first conjecture. Note that as defined in the theory, RWU is conceptually the superimposing of two processes: standard RWU, which creates SWP heterogeneity, and compensatory RWU, which dissipates (and is driven by) SWP heterogeneity but is independent of the plant's instantaneous transpiration rate.

Secondly, soil water flow is a process dissipating SWP heterogeneity; a high soil hydraulic conductivity thus favours SWP heterogeneity dissipation and leads to better predictions by approximations that use the first conjecture. Note that, even though silt loam hydraulic conductivity is mostly lower than that of sandy loam at the beginning of the simulations (see conductivity ranges in Fig. 3b), it stays relatively high at low soil matric potentials, which explains the higher accuracy of the silt loam than the sandy loam scenarios.

It is also worth noting that, in general, structural and parameterisation errors in a RWU model may have a limited impact on SWP distributions when soil water flow is a dominating process, as previously discussed by Hupet et al. (2002).

\subsubsection{Spatio-temporal distribution of processes: comparison with 1-D results}

This section clarifies the underlying assumption on soil water horizontal redistribution when using 1-D soil discretisation, and provides further insight on how it may impact model errors in space and time.

As shown in Fig. 6 for scenario "high $T_{\text {daily }}$ on silt loam", the intensity of each process redistributing water can be rated 
a)

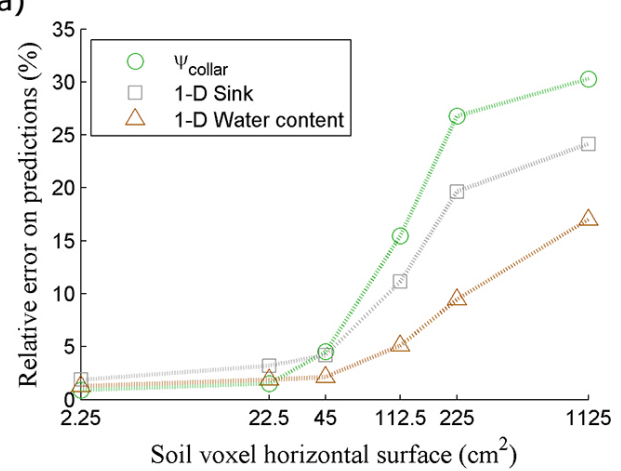

c)

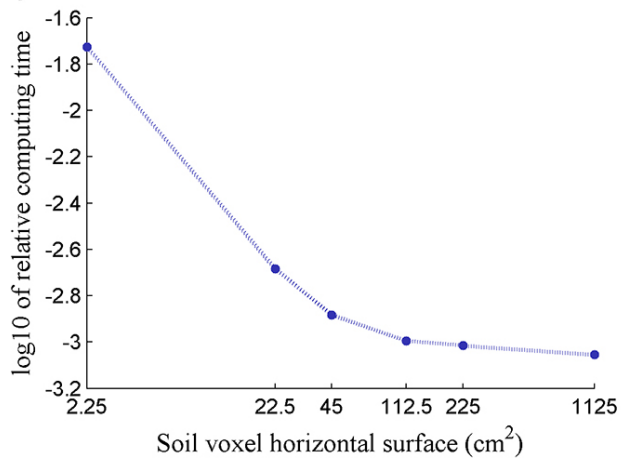

b)

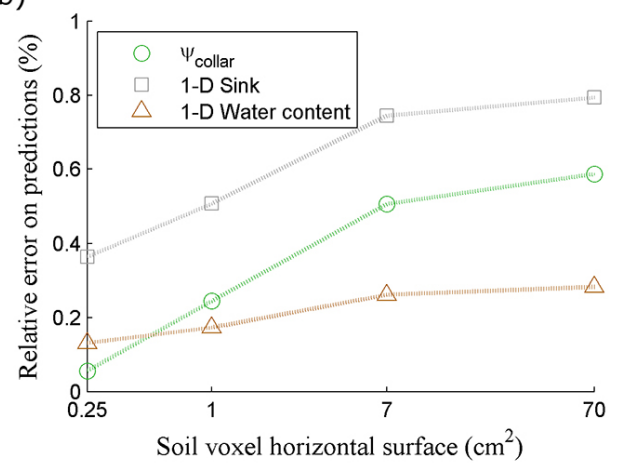

d)

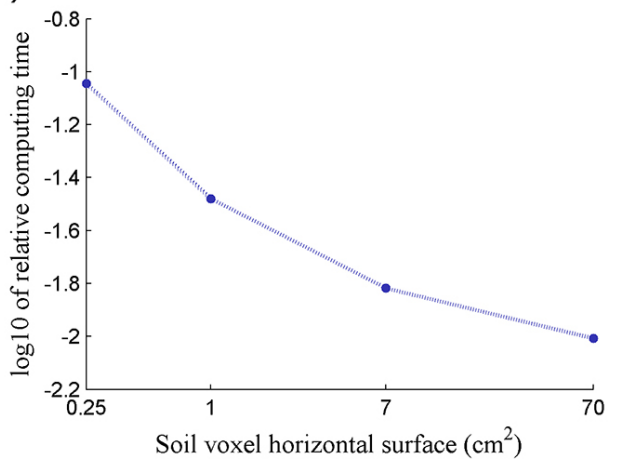

Fig. 5. Relative errors on three state variable predictions ( $\psi_{\text {collar }}, 1-\mathrm{D}$ sink and 1-D water content) when using upscaled soil elements whose inner SWP is considered as homogeneous, for maize (a) and winter wheat (b). Relative computing time for maize (c) and wheat (d). $x$ axes on logarithmic scale. Scenario: high $T_{\text {daily }}$ on silt loam.

in terms of its total positive volumetric divergence of water flow (total negative volumetric divergence being equivalent to the positive one, by definition, since these processes only redistribute water in the system). Blue lines correspond to processes as they occurred in the reference scenarios while the red ones are for 1-D scenarios. Solid and dotted lines correspond, respectively, to horizontal and vertical spatial components of the processes. Figure $6 \mathrm{a}$ and $\mathrm{c}$ shows water redistribution rates by soil, evolving with time, while Fig. $6 \mathrm{~b}$ and $\mathrm{d}$ shows water redistribution rates by roots. Eventually, Fig. $6 \mathrm{a}$ and $\mathrm{b}$ corresponds to maize, while Fig. $6 \mathrm{c}$ and $\mathrm{d}$ corresponds to winter wheat.

In Fig. 6a (maize), one can see that the assumed horizontal redistribution rate of water by soil in 1-D is overestimated during daytime; reference horizontal soil water flow is, thus, far from sustaining the necessary flow rate to keep SWP homogeneous. Also, during nighttime, even though decreased, reference horizontal soil water flow continues, due to the persistence of SWP horizontal heterogeneities, while in 1-D, the assumed horizontal water flow stops as soon as the plant stops transpiring (except once compensatory RWU significantly compensates vertical SWP heterogeneities at night). Conversely, in Fig. 6c (wheat), similar peaks of divergence of horizontal soil water flow can be noticed in both reference and 1-D scenarios. This can be attributed to the fact that water needs to flow on much shorter horizontal distances to compensate wheat SWP heterogeneities, and thus is much more effective in dissipating these heterogeneities (which almost disappear at night). For both maize and wheat, the vertical component of divergence of soil water flow is slightly underestimated in 1-D, which suggests that this process is affected by the hypothesis of horizontally homogeneous SWP, and may actually participate in dissipating SWP horizontal heterogeneities in reference scenarios.

For maize, both components of compensatory RWU are largely underestimated in 1-D (especially the horizontal one, which is null in 1-D, since SWP is considered as horizontally uniform), which is not the case for wheat, whose dominant vertical component of compensatory RWU is well represented in 1-D (see Fig. 6d).

During the second week of simulation, compensatory RWU rates reach increasingly high values (approximately 10 and $250 \mathrm{~cm}^{3}$ day $^{-1}$ redistributed in the profile, respectively for wheat and maize). For maize, compensatory RWU rates are similar or even higher than water redistribution rates by soil. Such integrated values of redistribution of water uptake are also non-negligible as compared to each plant's daily transpiration rate (respectively 27 and $600 \mathrm{~cm}^{3} \mathrm{~d}^{-1}$ ). This confirms that the process of compensatory RWU might have a major impact on plant water availability (Feddes et al., 

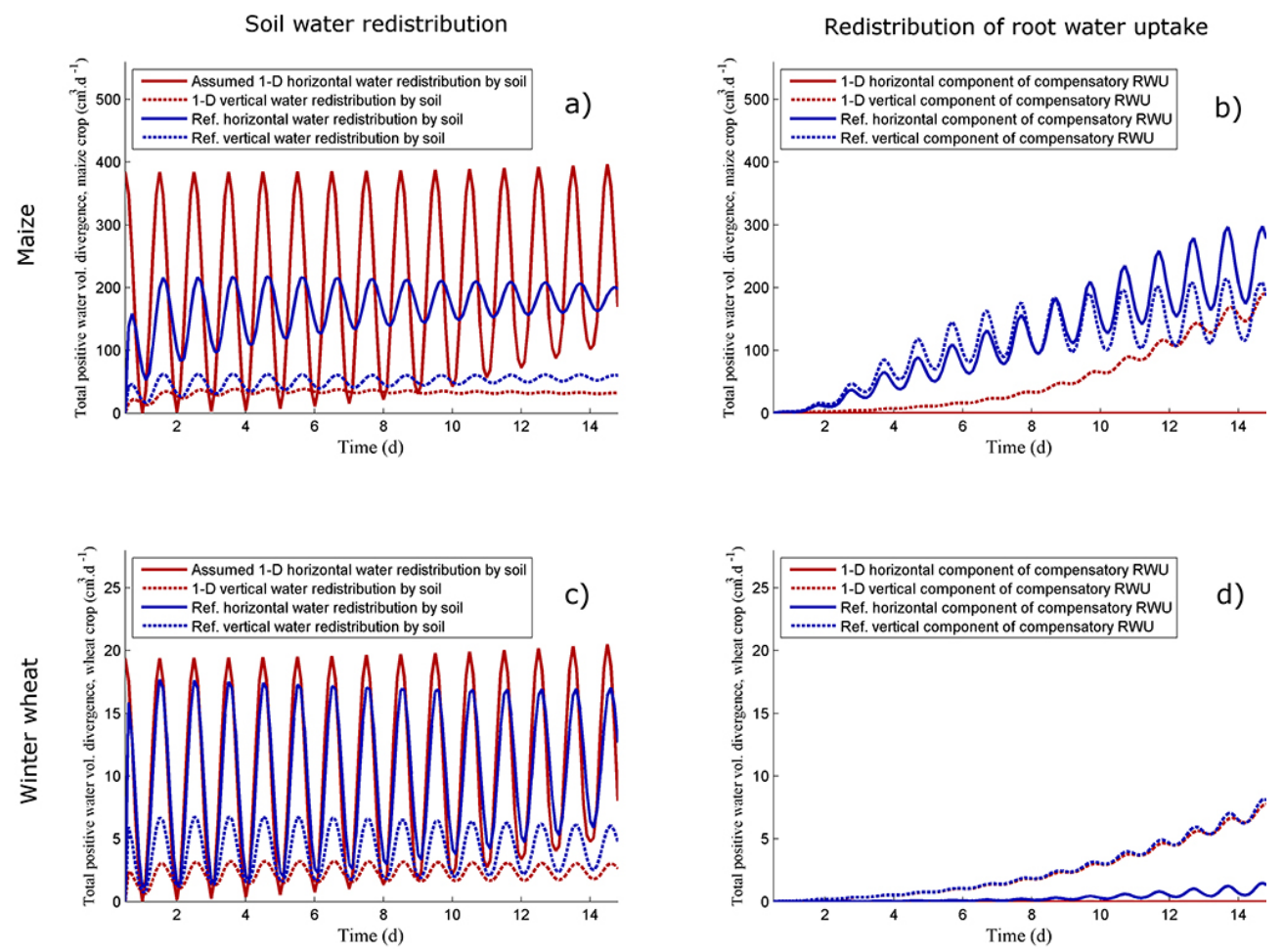

Fig. 6. Rating of processes dissipating soil water potential heterogeneity by (a, c) soil and (b, d) roots, in scenario "high $T_{\text {daily }}$ on silt loam", for $(\mathbf{a}, \mathbf{b})$ maize and $(\mathbf{c}, \mathbf{d})$ wheat.

2001; Teuling et al., 2006). However, compensatory RWU takes some time to become significant, as compared to horizontal and vertical water redistribution by soil. This can be explained by the fact that, while SWP heterogeneity increases with time, root system hydraulic conductances do not change; redistribution of water by the root system thus increases. At the same time, soil hydraulic conductivities tend to decrease (due to soil water content reduction); redistribution of water by soil capillary flow thus becomes of lesser importance as compared to compensatory RWU. That sort of reflection was previously raised by Gardner and Ehlig (1963), who stated that, with soil drying, "while processes such as capillary rise see their rate reduced, due to a decreased soil hydraulic diffusivity, an increasing proportion of water moves upward through roots, which somehow short-circuits the path of water movement through soil."

As illustrated in Fig. 6 (left subplots), vertical soil water redistribution was generally the least important process, in terms of rates; which can be explained by the fact that, on long vertical distances, equivalent soil hydraulic resistances are high enough to limit redistribution (water has to flow through a larger number of hydraulic resistances in series), and thus prevent SWP heterogeneities from being dissipated.

In case horizontal soil water flow would actually not be fast enough to equilibrate a layer's SWP, the assumed water potential at soil-root interfaces would be overestimated in 1-D. This is exactly the observed response in scenarios of
RWU by maize, where local SWP sensed by the plant decreases slower than in reference scenarios (Fig. 7a vs. 7d). This overestimation of local SWP sensed by the plant has two main consequences: (i) an underestimation of compensatory RWU (Fig. 7b vs. 7e, and dotted lines in Fig. 7f), and (ii) an overestimation of total SWP sensed by the plant (Fig. 7c) inducing underestimation of plant water stress (Fig. 7f).

It is notable that, for the same $T_{\text {act }}$, errors on $\psi_{\text {collar }}$ equal errors on $\psi_{\mathrm{s} \text { eq }}$ since the difference between these variables is $\frac{T_{\text {act }}}{K_{\text {rs }}}$, which has no spatial dimension, and thus, is not affected by a spatial dimension reduction. Also, values of compensatory RWU in Fig. 7b and e are given as fluxes per plant in soil layers of $1.5 \mathrm{~cm}$ height. As a matter of comparison, the spatial integration of positive terms is given in Fig. 7f, while the integration of all terms would be zero by definition.

Figure 8 is the equivalent of Fig. 7 for winter wheat on sandy loam instead of maize on silt loam. The 1-D system state appears to be very close to the reference one for all variables. Even though $\psi_{\text {collar }}$ and $\psi_{\text {s eq }}$ are slightly overestimated at night and underestimated during daytime, $T_{\text {act }}$ follows the same trend in both simulations. Conversely to results shown in Fig. 7, compensatory RWU is slightly overestimated in 1-D (see Fig. 8f), possibly due to water depletion around deep roots of wheat in the reference scenario, which limited the compensation rate. Proportionally to the total uptake rate, the compensation rate was always more intense on 

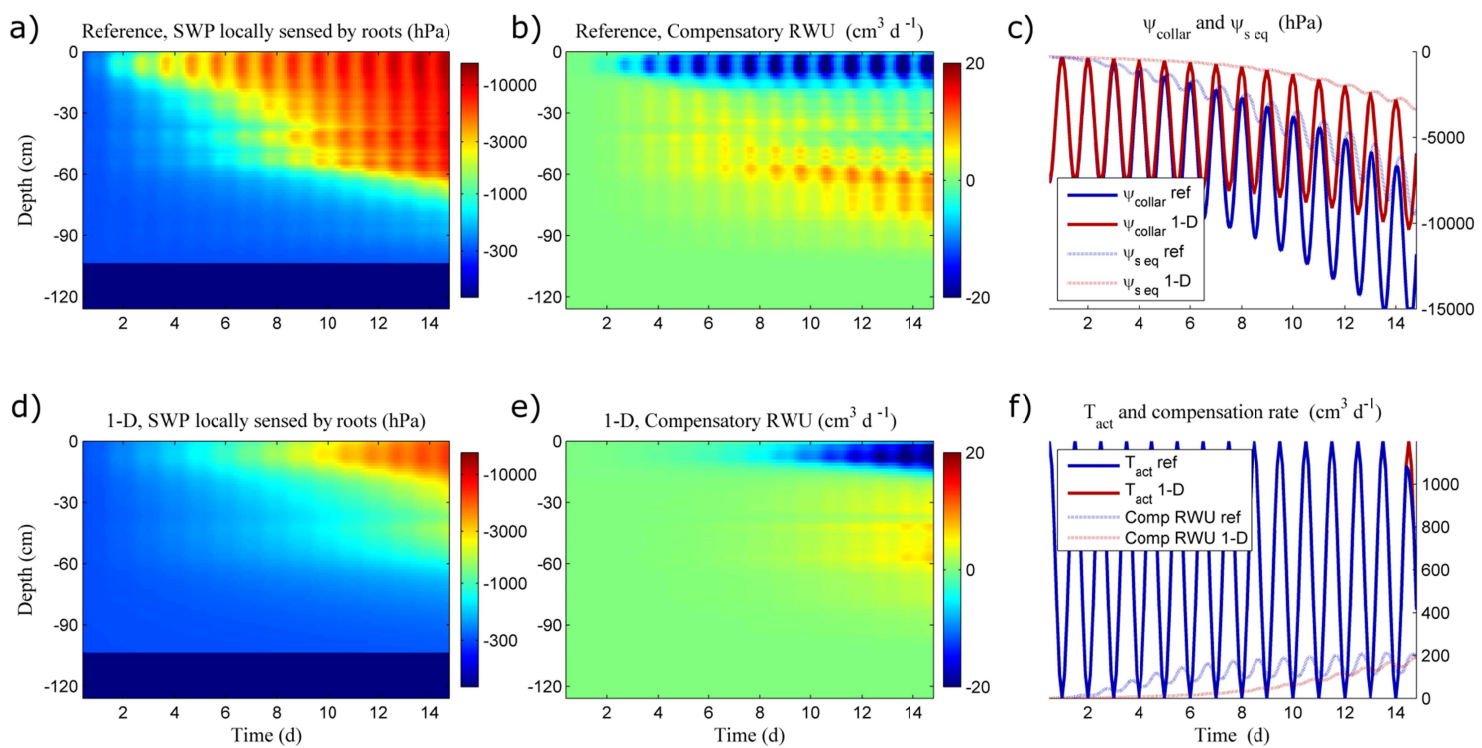

Fig. 7. Spatio-temporal distribution of (a, d) SWP locally sensed by roots and (b, e) compensatory RWU rates (spatial integration of positive terms), respectively in reference and 1-D scenarios. Temporal evolution of (c) plant collar water potential and SWP sensed by the plant, and (f) actual transpiration and compensation rates (scenario: maize, high $T_{\text {daily }}$ on silt loam).

sandy loam than on silt loam, seemingly because water is not as efficiently redistributed by the sandy soil.

A conclusion of the detailed comparison between 1-D and reference maize scenarios is that, when horizontal redistribution of water by soil is a limiting process, there is a clear need to account for differences between bulk SWP and water potential sensed by roots in soil layers, in order to avoid biased predictions of compensatory RWU and plant water stress, in dimensionally simplified soil-plant systems. A physical approach presented in Sect. 2.3 was developed by De Jong Van Lier et al. (2006) for that purpose, of which opportunities and limitations are discussed in the next section.

\subsection{Second conjecture: solution for water potential dif-} ferences between bulk soil and root surface in 1-D soil layers

In this section, limitations of the second conjecture and tested adaptations aiming at better accounting for unfulfilled assumptions are discussed.

\subsubsection{Horizontally heterogeneous rooting pattern}

Like macroscopic RWU models using a "microscopic approach" (Raats, 2007; De Jong Van Lier et al., 2008; Jarvis, 2011), the second conjecture allows predicting SWP variations between the bulk soil ( $\left.\psi_{\mathrm{s}} \mathrm{Up}\right)$ and soil-root interfaces $\left(\psi_{\text {sr Up }}\right)$ by assuming a horizontally homogeneous root distribution, which implies that the water dynamics around roots is the same (their properties being considered as identical).

Yet, for maize crops, due to the wide-row sowing pattern, two features are in contradiction with the second conjec- ture's assumptions: (i) water potentials at soil-root interfaces are not horizontally homogeneous (see for instance left subplot of Fig. 4), and (ii) the horizontal rooting pattern is not uniform. As demonstrated in Eq. (11), in each soil layer, a unique value of $\psi_{\mathrm{sr} \mathrm{Up}, g}$ may lead to the right average sink term for the layer. The microscopic approach might help finding this layer's "equivalent soil-root interface water potential", which makes it unnecessary to search for the full range of soil-root interface water potentials in each soil layer. The second contradiction is more of an issue since no definition of the geometrical factor $\rho$ (see Eq. B1 for its theoretical formulation) accounts for horizontal rooting pattern heterogeneity. However, knowing values of $S_{\mathrm{Up}, g}, M_{\mathrm{sr} \text { Up, } g}$ and $M_{\mathrm{S} \text { Up, } g}$ (from the reference scenarios), an effective value of $\rho_{g}$ was calculated at each depth for each time step of the scenarios, by using Eq. (17). As shown in Fig. 9a for scenario "maize high $T_{\text {daily }}$ on silt loam", the effective values grouped by depth are significantly lower than theoretical values of $\rho$ (blue dotted line), which means that the system behaves as if there were much fewer roots, or maybe, one "big root". This necessity to use smaller values of $\rho$ was already noticed in comparisons with experimental data, by Faria et al. (2010), who interpreted that feature as a consequence of rooting heterogeneity, poor contact at soil-root interfaces and inactivity of a significant percentage of roots (approximately $95 \%$ ), which thus should not be taken into account when calculating $\rho$. Through this modelling study, we investigated and confirmed the expected impact of horizontal rooting heterogeneity on $\rho$.

Note that since root geometry does not change during scenarios, effective $\rho$ values at a certain depth should 

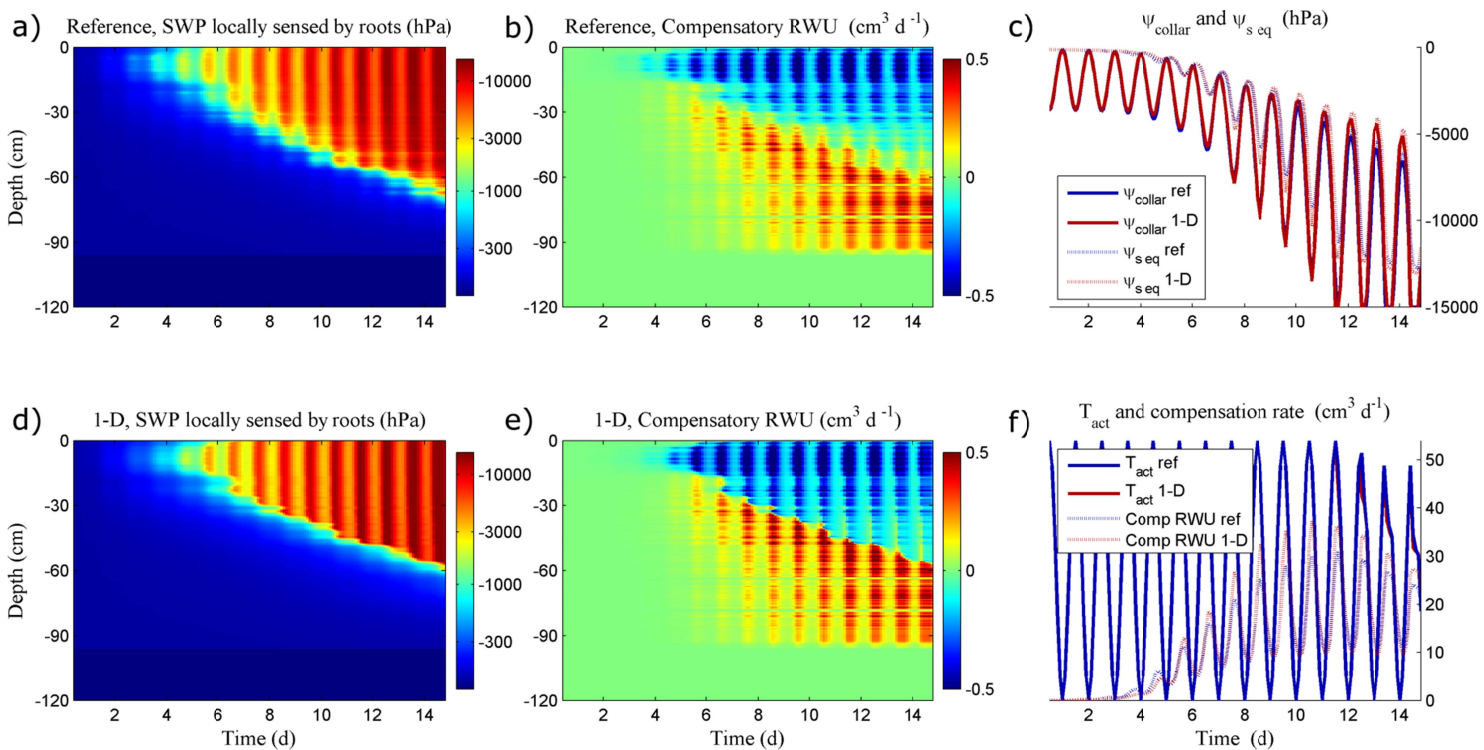

Fig. 8. Spatio-temporal distribution of (a, d) SWP locally sensed by roots and (b, e) compensatory RWU rates (spatial integration of positive terms), respectively in reference and 1-D scenarios. Temporal evolution of (c) plant collar water potential and SWP sensed by the plant, and (f) actual transpiration and compensation rates (scenario: winter wheat, high $T_{\text {daily }}$ on sandy loam).

theoretically remain constant with time. As shown in Fig. 9a, they actually cover a certain range of effective values, which are also strongly sensitive to soil type (not shown). One should thus be careful when using the theoretical parameterisation of $\rho$ for root systems with heterogeneous horizontal distribution.

Figure $9 \mathrm{c}$ shows the same comparison for wheat, whose theoretical $\rho$ values are much closer to the effective ones. This confirms that the theoretical parameterisation is more reliable for wheat, whose horizontal root distribution is indeed rather uniform.

Note that negative effective values of $\rho$ are not displayed in Fig. 9a and c. These however occur in reference simulations when roots exude water while $\psi_{\text {sr Up }}$ is still lower than the corresponding layer's bulk SWP. This transient situation cannot be predicted by the default model of water depletion around roots, since the geometrical factor $\rho$ is defined positive (see Eq. B1).

\subsubsection{Transient rate of root water uptake}

Another assumption of macroscopic RWU models using a "microscopic approach" to predict SWP depletion at soilroot interfaces is that rates of water uptake are constant with time. Water uptake rates at a soil-root interface change over time due to temporal changes in plant transpiration but also due to compensation mechanisms in the connected root system. Since the soil system has a memory due to its buffer capacity, the water potential profile around a root at a certain time does not depend only on the extraction rate at that time but also on previous extraction rates. Thus, using a weighted- mean of past sink terms in Eq. (17) rather than the sink term at a given moment might be better to predict the difference between soil-root interface $\psi_{\text {sr Up }}$ and bulk soil $\psi_{\mathrm{s} \text { Up }}$.

In this section, we tested if reference values of $M_{\mathrm{sr} \mathrm{Up}, g}$ (from which $\psi_{\mathrm{sr} \text { Up, } g}$ can directly be deduced) could be predicted from Eq. (17), either by using the theoretical values of $\rho_{g}$ and instantaneous $S_{\mathrm{Up}, g}$ ("default method"), or by using the mean values of effective $\rho_{g}$ (red vertical lines in the box plots in Fig. 9a and c) and instantaneous $S_{\mathrm{Up}, g}$ ("average $\rho$ " method), or eventually by using time-averaged values of $S_{\mathrm{Up}, g}$, in addition of the mean effective $\rho_{g}$ ("average $\rho \& S$ " method).

Figure $9 \mathrm{~b}$ shows the results obtained for maize at all timesteps of the "high $T_{\text {daily }}$ on silt loam" scenario. The "1:1 line" illustrates the position of the reference $M_{\mathrm{sr}} \mathrm{Up}, g$, while black circles correspond to the layer's bulk MFP (and to the $M_{\mathrm{sr} \text { Up, } g}$ predicted under the first conjecture). Mostly, even though more accurate than the first conjecture, using the "default method" (red crosses) still resulted in an overestimation of $M_{\mathrm{sr} \mathrm{Up}, g}$, mainly due to the theoretical overestimation of $\rho$. Effective methods "average $\rho$ " and "average $\rho \&$ $S$ " allowed increasing the accuracy of the predictions around the 1:1 line, however significant differences persist, mainly in dry conditions (where small errors on $M_{\mathrm{sr}} \mathrm{Up}, g$ moreover have a high impact on $\psi_{\mathrm{sr} \mathrm{Up}, g}$ ). The prediction of negative values of $M_{\mathrm{Sr}} \mathrm{Up}, g$ is also problematic since the function providing MFP values from soil matric potentials is positive by definition. Consequently, no $\psi_{\mathrm{sr} \text { Up, } g}$ value can be deduced from a negative $M_{\mathrm{sr} \text { Up, } g}$. Even though both effective methods were sensitive to the chosen averaging function, none of the tested functions allowed reaching satisfying results for 
a)

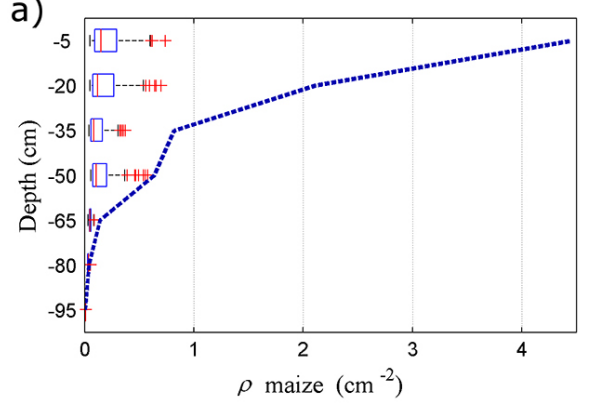

c)

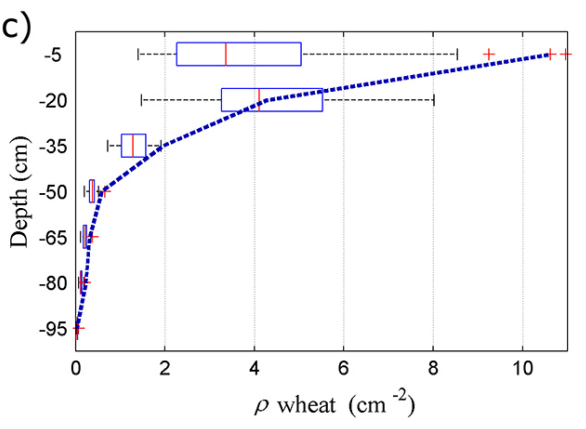

b)
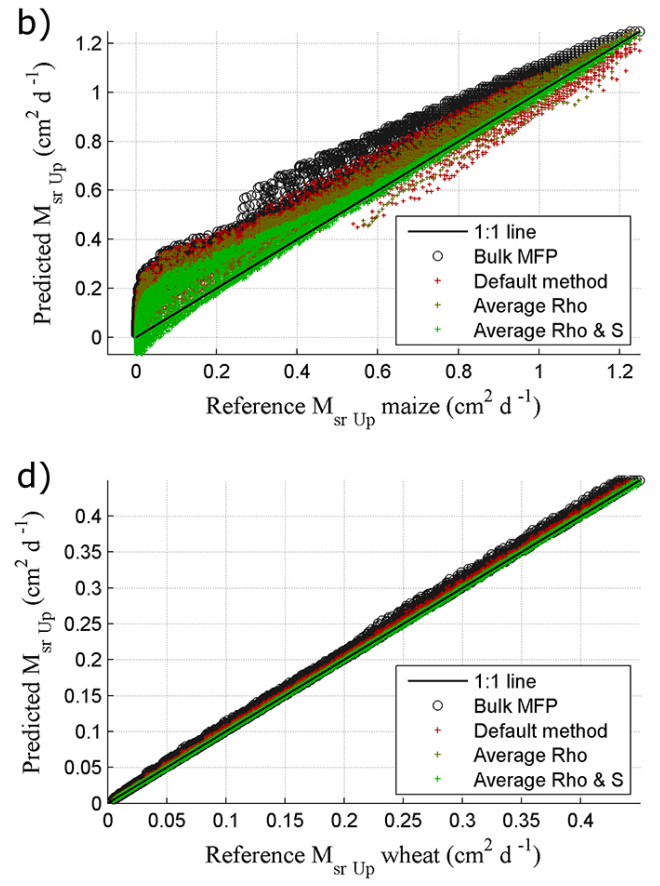

Fig. 9. System effective properties and state from the scenario "high $T_{\text {daily }}$ on silt loam". Theoretical (blue dotted lines) and effective (box plots) values of $\rho$ for maize (a) and wheat (c). The layer's matric flux potential at soil-root interfaces predicted using Eq. (17) with default and effective methods, compared with reference values, for maize (b) and wheat (d).

maize (averaging functions used for results shown in Fig. 9b and $\mathrm{d}$ : mean function for $\rho$, and $36 \mathrm{~h}$ average for the sink term). For wheat (Fig. 9d), results were already satisfying under the first conjecture, but could be improved by using the second conjecture, as shown by Fig. 9d.

When the RWU model using the second conjecture was further coupled to the Richards equation, the frequent prediction of negative values of $M_{\mathrm{sr}} \mathrm{Up}$ (happens when $M_{\mathrm{S} \mathrm{Up}, g}<$ $\frac{S_{\mathrm{Up}, g}}{\rho_{g}}$, typically when the soil becomes dry) and oscillating $\psi_{\text {sr Up, } g}$ caused non-convergence issues (mainly for simulations on sandy loam). These could not be solved in this study.

\subsubsection{Closing remarks on the second conjecture}

Ideally, exact physical expressions would allow accounting for transient RWU rates and heterogeneous rooting distribution, with a resulting model shape that would possibly have to be adapted as compared to Eqs. (17) and (B1). However, such opportunity does not exist today, and a simple alternative is to use effective parameters and variables such as described in previous paragraphs and suggested by Faria et al. (2010), even though they entail a loss of physical meaning of the model.

The proposed effective methods, accounting for unfulfilled assumptions of the De Jong Van Lier et al. (2006) model, did not allow significantly improving predictions of differences between bulk SWP and SWP sensed by roots for 1-D spatial discretisation, except in conditions in which the first conjecture was already satisfying (winter wheat crop on silt loam). There is however a clear need for accurate functions predicting soil-root interface water potential, in order to correctly predict compensatory RWU and plant water stress. In the future, that problem might be solved through the development of specific analytical solutions for each type of system properties.

\section{Conclusions and outlook}

The objective of this paper was to provide a theoretical framework and exploratory analysis regarding the use of "upscaled" RWU models, partly or fully neglecting SWP horizontal heterogeneity within the root zone. We demonstrated how to derive upscaled RWU parameters and state variables (among which the upscaled soil-root interface water potential) from small scale information. Two simplified approaches aiming at estimating such upscaled water potential (when small-scale information is not available) were then tested in soil-plant hydrodynamics scenarios, for two crops with rather heterogeneous (maize) or homogeneous (winter wheat) horizontal rooting distributions.

With the first approach, SWP was considered as homogeneous in upscaled soil elements. For maize, neglecting SWP heterogeneities in the direction of the row was shown to be a good compromise between accuracy (relative errors mostly below $5 \%$ ) and computing time (reduced by $67-96 \%$ ). 
However, in 1-D, the assumed horizontal water redistribution rate by soil was far above reference 3-D values during daytime and far below them at night. Consequently, the intensity of compensatory RWU was underestimated while plant collar water potential was overestimated. For winter wheat, the rather uniform rooting distribution tended to generate shortdistance SWP heterogeneities, and favoured a fast horizontal redistribution of water by soil. Therefore, 1-D processes of water redistribution were in agreement with reference values (relative errors mostly below $5 \%$ ), and computation time could be reduced by $80-99 \%$. More generally, the accuracy of the first approach was improved when processes creating SWP heterogeneity were reduced (e.g. low plant transpiration rate) and processes dissipating SWP heterogeneity were dominant (e.g. high soil hydraulic conductivity). A conclusion of the first conjecture is that a 1-D soil geometry is enough to represent soil-plant water dynamics for winter wheat, but not for maize. Representing the latter case in 1-D would require accounting for water depletion around roots, which is the aim of the second conjecture.

With the second conjecture, the difference between bulk SWP and SWP sensed by roots in 1-D soil layers was estimated with an approximate analytical solution of soil water flow towards roots. The validity of the latter model, when two of its assumptions are not met (regular rooting distribution and constant RWU rate) was questioned. First, horizontal rooting heterogeneity was shown to impact effective values of the geometrical parameter $\rho$ for maize, while a better agreement between theoretical and effective values of $\rho$ were noticed for the rather regular rooting distribution of winter wheat. Second, accounting for past uptake rates over a time window of $36 \mathrm{~h}$ improved the agreement with reference results, whose local RWU rates were transient. However, for maize, the layers' soil-root interface water potentials could not be accurately predicted, especially in dry conditions.
This study confirmed that the use of 1-D spatial discretisation to represent soil-plant water dynamics is a worthy choice for densely seeded crops. It also highlighted that, for wide-row crops, further theoretical developments, better accounting for actual system properties, might be needed to properly predict plant collar water potential and compensatory RWU, as compared to fine-scale simulations.

Future prospects in line with this study could also focus on the analysis of implications of using even coarser grids when modelling soil-plant hydrodynamics at the plot or larger scales.

Acknowledgements. During the preparation of this manuscript, V. Couvreur was supported by the Fonds National de la Recherche Scientifique (FNRS) of Belgium as a research fellow, by the Belgian American Educational Foundation (BAEF), as UCLouvain fellow, and by the Wallonie-Bruxelles International (WBI) with a WBI.WORLD excellence grant. The authors thank these funding agencies for their financial support as well as Quirijn de Jong van Lier and an anonymous referee for their constructive comments. This work is a contribution of the Transregio Collaborative Research Center 32, Patterns in Soil-Vegetation-Atmosphere Systems: Monitoring, Modelling and Data Assimilation, which is funded by the German research association, DFG.

Edited by: I. Neuweiler 


\section{Appendix A}

Definition of soil water flow divergence necessary to keep soil water potential homogeneous during root water uptake in upscaled soil elements

From an initially uniform distribution of SWP inside a horizontally upscaled soil element, taking up a flux " $S_{\mathrm{Up}, g} . V_{\mathrm{Up}, g}$ " of water would generate SWP heterogeneity around roots if water was not redistributed. Leading SWP to a new homogeneous state inside the upscaled soil element instantly requires a horizontal divergence of soil water flow (mostly negative in regions where RWU occurs), which depends on the characteristic distribution of RWU inside the upscaled soil element.

When using upscaled soil elements, one indirectly assumes that the element is an entity keeping its inner water potential homogeneous, independently of other upscaled elements. In other words, the equilibration of inner SWP requires soil water redistribution, which is assumed to come from the inside of the upscaled element only. The divergence of soil capillary flow over the upscaled soil element is thus zero regarding the equilibration step, while divergences may locally be different from zero in its constituting elements. Note that when calculating soil water flow between different upscaled soil elements, their divergence of water flow may of course be different from zero.

The following forms of the Richards equation thus apply, respectively for upscaled and fine soil elements, regarding the instantaneous equilibration of upscaled elements inner SWP:

$\frac{\partial \theta_{\mathrm{Up}, g}}{\partial t}=-S_{\mathrm{Up}, g}$,

$\frac{\partial \theta_{k}}{\partial t}=-\operatorname{Div}_{k}-S_{k}$

where $\operatorname{Div}_{k}\left(\mathrm{~L}^{3} \mathrm{~L}^{-3} \mathrm{~T}^{-1}\right)$ is the divergence of soil water flow in the $k$ th fine element, more commonly expressed as " $-\nabla$. $\left[K\left(\psi_{\mathrm{m}, k}\right) \nabla \psi_{\mathrm{s}, k}\right] ”$.

In order to keep SWP horizontally homogeneous inside an upscaled element (and considering soil hydraulic properties as uniform), all local $\frac{\partial \theta_{k}}{\partial t}$ need to equal $\frac{\partial \theta_{\mathrm{Up}, g}}{\partial t}$. From Eqs. (A1) and (A2) we thus obtain

$\operatorname{Div}_{k}=S_{U p, g}-S_{k}$.
Considering initial SWP as homogeneous inside the upscaled soil element, local uptake rates can be defined as standard fractions of the total uptake rate of the upscaled element:

$S_{k} \cdot V_{k}=S_{\mathrm{Up}, g} \cdot V_{\mathrm{Up}, g} \cdot \frac{\mathrm{SSF}_{k}}{\mathrm{SSF}_{\mathrm{Up}, g}}$,

where $\sum_{k=1}^{M} \varepsilon_{k, g} \cdot \mathrm{SSF}_{k}=\mathrm{SSF}_{\mathrm{Up}, g}$.

From Eqs. (A3) and (A4), the local divergence of soil water flow can be defined as follows:

$\operatorname{Div}_{k}=S_{\mathrm{Up}, g} \cdot \frac{V_{\mathrm{Up}, g}}{V_{k}} \cdot\left(\frac{V_{k}}{V_{\mathrm{Up}, g}}-\frac{\mathrm{SSF}_{k}}{\mathrm{SSF}_{\mathrm{Up}, g}}\right)$.

Since in our case, soil water flow divergence is simply a redistribution of water inside the upscaled element, the volumetric integration of positive terms equals that of negative terms, and half the volumetric integration of all absolute terms. We thus obtain the following definition of the volumetric integration of positive water flow divergence necessary to keep SWP uniform inside an upscaled soil element, $R_{\text {soil } \leftrightarrow \text { hyp }, g}\left(\mathrm{~L}^{3} \mathrm{~T}^{-1}\right)$ :

$R_{\text {Soil } \leftrightarrow \mathrm{hyp}, g}=\frac{\sum_{k=1}^{M} \varepsilon_{k, g} \cdot V_{k} \cdot\left|\operatorname{Div}_{k}\right|}{2}$
$=\left|S_{\mathrm{Up}, g}\right| \cdot V_{\mathrm{Up}, g} \cdot \frac{\sum_{k=1}^{M} \varepsilon_{k, g} \cdot\left|\frac{\mathrm{SSF}_{k}}{\operatorname{SSF}_{\mathrm{Up}, g}}-\frac{V_{k}}{V_{\mathrm{Up}, g}}\right|}{2}$.

Note that soil water flow divergence at scales lower than the scale of fine elements is not considered in the latter equation.

The coefficient $\frac{\sum_{k=1}^{M} \varepsilon_{k, g} \cdot\left|\frac{\mathrm{SSF}_{k}}{\mathrm{SSF}_{\mathrm{Up}, g}}-\frac{V_{k}}{V_{\mathrm{Up}, g}}\right|}{2}$ appears to be an indicator of how "generator of SWP heterogeneity" a HA is, inside an upscaled soil element (which could be enlarged up to the whole soil domain). Its value tends to zero for uniform standard sink distributions inside the upscaled element, which do not create SWP heterogeneities, and tends to one for a single root inside an infinitesimal part of the upscaled element, which corresponds to the case generating the biggest amount of heterogeneity for a given water uptake or exudation rate. 


\section{Appendix B}

Theoretical equation for the geometrical parameter $\rho_{g}$ for regular root distribution in a soil layer

De Jong Van Lier et al. (2006) provides the following theoretical equation for the geometrical parameter $\rho_{g}$ for regular root distribution in a soil layer:

$$
\rho_{g}=\frac{4}{r_{0, g}^{2}-\frac{a^{2}}{\pi \cdot \mathrm{RLD}_{g}}+2 \cdot\left(\frac{1}{\pi \cdot \mathrm{RLD}_{g}}+r_{0, g}^{2}\right) \cdot \ln \left(\frac{a}{r_{0, g} \cdot \sqrt{\pi \cdot \mathrm{RLD}_{g}}}\right)}
$$

where $r_{0, g}(\mathrm{~L})$ is the mean roots radius at the $g$ th depth, $a(-)$ is a parameter considered as equal to 0.53 (De Jong Van Lier et al., 2006), and $\mathrm{RLD}_{g}\left(\mathrm{~L}^{-2}\right)$ is the root length density at the $g$ th depth. 


\section{Appendix C}

\section{Equations for vertical and horizontal water redistribution rates by soil and roots}

Vertical and horizontal water redistribution rates by soil were calculated as the volumetric integration of the corresponding absolute components of water flow divergence between soil elements:

$R_{\mathrm{soil} \leftrightarrow}=\frac{1}{2} \cdot \sum_{k=1}^{M} \mid\left(J_{\mathrm{x} 2, k}-J_{\mathrm{x} 1, k}\right) \cdot d_{\mathrm{y}} \cdot d_{\mathrm{z}}$

$+\left(J_{\mathrm{y} 2, k}-J_{\mathrm{y} 1, k}\right) \cdot d_{\mathrm{x}} \cdot d_{\mathrm{z}}$

$R_{\mathrm{soil} \downarrow}=\frac{1}{2} \cdot \sum_{k=1}^{M}\left|\left(J_{\mathrm{z} 2, k}-J_{\mathrm{z} 1, k}\right) \cdot d_{\mathrm{x}} \cdot d_{\mathrm{y}}\right|$

where $R_{\text {soil } \leftrightarrow}$ and $R_{\text {soil } \downarrow}\left(\mathrm{L}^{3} \mathrm{~T}^{-1}\right)$ are, respectively, the horizontal and vertical components of water redistribution rates by soil, $J_{\mathrm{x} 1, k}$ and $J_{\mathrm{x} 2, k}\left(\mathrm{~L} \mathrm{~T}^{-1}\right)$ are soil water flow densities in the $x$ direction, respectively on the first and second side of soil element \# $k$, and $d_{\mathrm{x}}(\mathrm{L})$ is the length of soil elements in the $x$ direction (same logic for $y$ and $z$ directions).

Even though RWU rates have no direction per se, water redistribution between layers was considered vertical while redistribution resulting from horizontal heterogeneities was considered horizontal.
Vertical water redistribution rates by roots were calculated as the integration of absolute net compensatory RWU of each soil layer:

$R_{\text {root } \uparrow}=\frac{1}{2} \cdot \sum_{l=1}^{L}\left|\sum_{k=1}^{M} \varepsilon_{k, l} \cdot \beta_{k}\right|$

where $R_{\text {root } \downarrow}\left(\mathrm{L}^{3} \mathrm{~T}^{-1}\right)$ is the vertical water redistribution rate by roots, $\beta_{k}=S_{k} \cdot V_{k}-\mathrm{SSF}_{k} \cdot T_{\text {act }}\left(\mathrm{L}^{3} \mathrm{~T}^{-1}\right)$ is the compensatory RWU in the $k$ th soil element, $l(-)$ is the soil layer index, $L$ is the total number of soil layers, and $\varepsilon_{k, l}(-)$ equals 1 when the $k$ th soil element is included in the $l$ th soil layer and equals 0 otherwise.

Horizontal water redistribution rates by roots were calculated as the integration of absolute deviations of compensatory RWU as compared to the expected distribution of layers net compensatory RWU for horizontally uniform SWP:

$R_{\mathrm{root} \leftrightarrow}=\sum_{l=1}^{L} \frac{1}{2} \cdot\left(\sum_{k=1}^{M} \varepsilon_{k, l} \cdot\left|\beta_{k}-\frac{S S F_{k}}{\sum_{k=1}^{M} \varepsilon_{k, l} \cdot \mathrm{SSF}_{k}} \cdot\left(\sum_{k=1}^{M} \varepsilon_{k, l} \cdot \beta_{k}\right)\right|\right)$

where $R_{\text {root } \leftrightarrow}\left(\mathrm{L}^{3} \mathrm{~T}^{-1}\right)$ is the horizontal water redistribution rate by roots, $\sum_{k=1}^{M} \varepsilon_{k, l} \cdot \beta_{k}\left(\mathrm{~L}^{3} \mathrm{~T}^{-1}\right)$ is the net compensatory RWU in the $l$ th soil layer, $\frac{\operatorname{SSF}_{k}}{\sum_{k=1}^{M} \varepsilon_{k, l} \cdot \operatorname{SSF}_{k}}(-)$ is the fraction of net compensatory RWU expected in the $k$ th soil element in case SWP would be horizontally uniform in the $l$ th soil layer. 


\section{References}

Allen, R. G., Pereira, L. S., Raes, D., and Smith, M.: Crop evapotranspiration - Guidelines for computing crop water requirements, FAO Irrigation and drainage, Paper 56, Rome, 1998.

Beff, L., Günther, T., Vandoorne, B., Couvreur, V., and Javaux, M.: Three-dimensional monitoring of soil water content in a maize field using Electrical Resistivity Tomography, Hydrol. Earth Syst. Sci., 17, 595-609, doi:10.5194/hess-17-595-2013, 2013.

Bramley, H., Turner, N. C., Turner, D. W., and Tyerman, S. D.: Comparison between gradient-dependent hydraulic conductivities of roots using the root pressure probe: the role of pressure propagations and implications for the relative roles of parallel radial pathways, Plant Cell Environ., 30, 861-874, 2007.

Bramley, H., Turner, N. C., Turner, D. W., and Tyerman, S. D.: Roles of morphology, anatomy, and aquaporins in determining contrasting hydraulic behavior of roots, Plant Physiol., 150, 348364, 2009.

Carsel, R. F. and Parrish, R. S.: Developping joint probabilitydistributions of soil-water retention characteristics, Water Resour. Res., 24, 195-200, 1988.

Couvreur, V., Vanderborght, J., and Javaux, M.: A simple threedimensional macroscopic root water uptake model based on the hydraulic architecture approach, Hydrol. Earth Syst. Sci., 16, 2957-2971, doi:10.5194/hess-16-2957-2012, 2012.

De Jong Van Lier, Q., Metselaar, K., and Van Dam, J. C.: Root water extraction and limiting soil hydraulic conditions estimated by numerical simulation, Vadose Zone J., 5, 1264-1277, 2006.

De Jong Van Lier, Q., Van Dam, J. C., Metselaar, K., De Jong, R., and Duijnisveld, W. H. M.: Macroscopic root water uptake distribution using a matric flux potential approach, Vadose Zone J., 7, 1065-1078, 2008.

Domec, J. C. and Pruyn, M. L.: Bole girdling affects metabolic properties and root, trunk and branch hydraulics of young ponderosa pine trees, Tree Physiol., 28, 1493-1504, 2008.

Doussan, C., Pages, L., and Vercambre, G.: Modelling of the hydraulic architecture of root systems: An integrated approach to water absorption - Model description, Ann. Bot.-London, 81, 213-223, 1998.

Doussan, C., Pierret, A., Garrigues, E., and Pages, L.: Water uptake by plant roots: II - Modelling of water transfer in the soil rootsystem with explicit account of flow within the root system Comparison with experiments, Plant. Soil, 283, 99-117, 2006.

Draye, X., Kim, Y., Lobet, G., and Javaux, M.: Model-assisted integration of physiological and environmental constraints affecting the dynamic and spatial patterns of root water uptake from soils, J. Exp. Bot., 61, 2145-2155, 2010.

Durigon, A., dos Santos, M. A., van Lier, Q. D., and Metselaar, K.: Pressure Heads and Simulated Water Uptake Patterns for a Severely Stressed Bean Crop, Vadose Zone J., 11, 14 pp., doi:10.2136/vzj2011.0187, 2012.

Faria, L. N., Da Rocha, M. G., Van Lier, Q. D., and Casaroli, D.: A split-pot experiment with sorghum to test a root water uptake partitioning model, Plant. Soil, 331, 299-311, 2010.

Feddes, R. A., Kowalik, P., Kolinska-Malinka, K., and Zaradny, H.: Simulation of field water uptake by plants using a soil water dependent root extraction function, J. Hydrol., 31, 13-26, 1976.
Feddes, R. A., Hoff, H., Bruen, M., Dawson, T., De Rosnay, P., Dirmeyer, P., Jackson, R. B., Kabat, P., Kleidon, A., Lilly, A., and Pitman, A. J.: Modeling root water uptake in hydrological and climate models, B. Am. Meteorol. Soc., 82, 2797-2809, 2001.

Frensch, J. and Steudle, E.: Axial and radial hydraulic resistance to roots of maize (Zea-mays-L), Plant Physiol., 91, 719-726, 1989.

Gardner, W. R.: Dynamic aspects of water availability to plants, Soil. Sci., 89, 63-73, 1960.

Gardner, W. R. and Ehlig, C. F.: The influence of soil water on transpiration by plants, J. Geophys. Res., 68, 5719-5724, 1963.

Hupet, F., Lambot, S., Javaux, M., and Vanclooster, M.: On the identification of macroscopic root water uptake parameters from soil water content observations, Water Resour. Res., 38, 1-14, 2002.

Jarvis, N. J.: Simple physics-based models of compensatory plant water uptake: concepts and eco-hydrological consequences, Hydrol. Earth Syst. Sci., 15, 3431-3446, doi:10.5194/hess-15-34312011, 2011.

Javaux, M., Schroder, T., Vanderborght, J., and Vereecken, H.: Use of a three-dimensional detailed modeling approach for predicting root water uptake, Vadose Zone J., 7, 1079-1088, 2008.

Javaux, M., Couvreur, V., Vanderborght, J., and Vereecken, H.: Root Water Uptake: From 3D Biophysical Processes to Macroscopic Modeling Approaches, Vadose Zone J., 16 pp., doi:10.2136/vzj2013.02.0042, 2013.

Jolliet, O. and Bailey, B. J.: The effect of climate on tomato transpiration in greenhouses: measurements and models comparison, Agr. Forest Meteorol., 58, 43-62, 1992.

Manabe, S.: Climate and ocean circulation .I. Atmospheric circulation and hydrology of earth surface, Mon. Weather Rev., 97, 739-774, 1969.

Pages, L., Vercambre, G., Drouet, J. L., Lecompte, F., Collet, C., and Le Bot, J.: Root Typ: a generic model to depict and analyse the root system architecture, Plant Soil, 258, 103-119, 2004.

Raats, P. A. C.: Uptake of water from soils by plant roots, Transport Porous Med., 68, 5-28, 2007.

Sanderson, J., Whitbread, F. C., and Clarkson, D. T.: Persistent Xylem Cross-Walls Reduce The Axial Hydraulic Conductivity In The Apical $20 \mathrm{Cm}$ Of Barley Seminal Root Axes - Implications For The Driving Force For Water-Movement, Plant Cell Environ., 11, 247-256, 1988.

Schneider, C. L., Attinger, S., Delfs, J.-O., and Hildebrandt, A.: Implementing small scale processes at the soil-plant interface - the role of root architectures for calculating root water uptake profiles, Hydrol. Earth Syst. Sci., 14, 279-289, doi:10.5194/hess14-279-2010, 2010.

Schroeder, T., Javaux, M., Vanderborght, J., and Vereecken, H.: Comment on "Root water extraction and limiting soil hydraulic conditions estimated by numerical simulation", Vadose Zone J., 6, 524-526, 2007.

Schroeder, T., Javaux, M., Vanderborght, J., Korfgen, B., and Vereecken, H.: Implementation of a Microscopic Soil-Root Hydraulic Conductivity Drop Function in a Three-Dimensional Soil-Root Architecture Water Transfer Model, Vadose Zone J., 8, 783-792, 2009a.

Schroeder, T., Tang, L., Javaux, M., Vanderborght, J., Körfgen, B., and Vereecken, H.: A grid refinement approach for a threedimensional soil-root water transfer model, Water Resour. Res., 45, W10412, doi:10.1029/2009WR007873, 2009b. 
Sperling, O., Shapira, O., Cohen, S., Tripler, E., Schwartz, A., and Lazarovitch, N.: Estimating sap flux densities in date palm trees using the heat dissipation method and weighing lysimeters, Tree Physiol., 32, 1171-1178, 2012.

Tardieu, F. and Simonneau, T.: Variability among species of stomatal control under fluctuating soil water status and evaporative demand: modelling isohydric and anisohydric behaviours, J. Exp. Bot., 49, 419-432, doi:10.1093/jexbot/49.suppl_1.419, 1998.

Tazawa, M., Ohkuma, E., Shibasaka, M., and Nakashima, S.: Mercurial-sensitive water transport in barley roots, J. Plant Res., 110, 435-442, 1997.

Teuling, A. J., Uijlenhoet, R., Hupet, F., and Troch, P. A.: Impact of plant water uptake strategy on soil moisture and evapotranspiration dynamics during drydown, Geophys. Res. Lett., 33, L03401, doi:10.1029/2005GL025019, 2006.
Van Genuchten, M. T.: A closed form equation for predicting the hydraulic conductivity of unsaturated soils, Soil Sci. Soc. Am. J., 44, 892-898, 1980.

Van Noordwijk, M. and De Willigen, P.: Agricultural concepts of roots: From morphogenetic to functional equilibrium between root and shoot growth, Neth. J. Agric. Sci., 35, 487-496, 1987.

Watt, M., Magee, L. J., and McCully, M. E.: Types, structure and potential for axial water flow in the deepest roots of field-grown cereals, New Phytol., 178, 135-146, 2008.

Weaver, J. E., Kramer, J., and Reed, M.: Development of root and shoot of winter wheat under field environment, Ecology, 5, 26$50,1924$. 